\title{
Traffic-aware Two-stage Queueing Communication Networks: Queue Analysis and Energy Saving
}

\author{
Nan Qi, Nikolaos I. Miridakis, Ming Xiao, Senior Member, IEEE, Theodoros A. Tsiftsis, Senior Member, IEEE, \\ Rugui Yao, Senior Member, IEEE, and Shi Jin, Senior Member, IEEE
}

\begin{abstract}
To boost energy saving for the general delay-tolerant IoT networks, a two-stage, and single-relay queueing communication scheme is investigated. Concretely, a traffic-aware $N$ threshold and gated-service policy are applied at the relay. As two fundamental and significant performance metrics, the mean waiting time and long-term expected power consumption are explicitly derived and related with the queueing and service parameters, such as packet arrival rate, service threshold and channel statistics. Besides, we take into account the electrical circuit energy consumptions when the relay server and access point (AP) are in different modes and energy costs for mode transitions, whereby the power consumption model is more practical. The expected power minimization problem under the mean waiting time constraint is formulated. Tight closed-form bounds are adopted to obtain tractable analytical formulae with less computational complexity. The optimal energy-saving service threshold that can flexibly adjust to packet arrival rate is determined. In addition, numerical results reveal that: 1) sacrificing the mean waiting time not necessarily facilitates power savings; 2) a higher arrival rate leads to a greater optimal service threshold; and 3) our policy performs better than the current state-of-the-art.
\end{abstract}

Index Terms-Delay tolerant IoT relaying networks, mean waiting time, $N$-threshold and gated policy, two-hop queueing, and traffic-aware power saving.

\section{INTRODUCTION}

\section{A. Background}

As wireless networks consecutively evolve, the Internet of Things (IoT), e.g., Machine-to-machine (M2M) and device to device (D2D) networks, is emerging as a promising network formation to fulfill the rising wireless network traffic demand worldwide [2]. IoT networks enable devices built-in sensors

Nan Qi is with the Key Laboratory of Dynamic Cognitive System of Electromagnetic Spectrum Space, Ministry of Industry and Information Technology, Nanjing University of Aeronautics and Astronautics, Nanjing, China, 210016. Nan Qi is also with National Mobile Communications Research Laboratory, Southeast University, Nanjing 210096, P. R. China (e-mail: nanqi.commun@gmail.com).

N. I. Miridakis is with the School of Electrical and Information Engineering and the Institute of Physical Internet, Jinan University, Zhuhai 519070, China and with the Department of Electrical and Electronic Engineering, University of West Attica, Aegaleo 12244, Greece (e-mail: nikozm@uniwa.gr).

Ming Xiao is with the School of Electrical Engineering of KTH, Royal Institute of Technology, Stockholm, Sweden (e-mail: mingx@kth.se).

T. A. Tsiftsis is with the School of Intelligence Science and Engineering, Jinan University, Zhuhai 519070, China (e-mail: theo_tsiftsis@jnu.edu.cn).

Rugui Yao is with the School of Electronics and Information, Northwestern Polytechnical University, China (e-mail: yaorg@nwpu.edu.cn)

Shi Jin is with the National Mobile Communications Research Lab, Southeast University, Nanjing, China (e-mail: jinshi@seu.edu.cn).

This work was supported in part by the National Natural Science Foundation of China (No. 61801218, 61871327). Part of this work has been presented in IEEE WCNC workshop 2019 [1]. to be connected to an IoT platform and integrates packets from different devices, therefore, devices can communicate with each other. Low-cost and low-energy-consumption IoT radio sensors find wide applications, e.g., smart farm/industrial monitor. With a rapid increase in the number of connected devices ( 25 billion by 2020 [3]), the energy consumption in the overall IoT networks dramatically increases and becomes a great concern. It is demonstrated that massive connected devices consume more power than conventional cellular and Wi-Fi networks [4]. IoT devices are mostly small-scale and powered by energy-constrained batteries. Therefore, a lowpower communication policy is crucial for IoT networks.

Note that IoT end-users may be placed in remote or human hard-to-reach areas, where there is no reliable transmission link. As such, small-scale short-range IoT devices are unable to transmit over a long distance (e.g., several kilometers), which means that there are difficulties in communicating with a distant AP. To enlarge the communication region, relay deployment has been widely proposed, especially for endusers with small transmitting power or being blocked by physical obstacles [4]-[7]. IoT relays gather data from users and relay it to destinations. As one example, M2M relaying has been proposed as a heterogeneous architecture for the European Telecommunications Standards Institute (ETSI) M2M and IEEE 802.15.4/802.16p IoT architectures [4]. Demos on mobile relay architectures for low-power IoT devices were presented in [5], [6]. The authors in [5] presented a relay-assisted video streaming application. In [6], the authors investigated a 5G IoT scenario for sensor data collection via drone relays. Therein, a relaying system was applied for environmental monitoring and agricultural applications. Compared with the ordinary IoT devices, IoT relays and access points (APs) have more resources but are more energy-consuming. That is, wide relay utilizations potentially lead to remarkably increasing global energy consumption [8].

Several prior research works investigated energy saving procedures that allow APs to dynamically adjust transmission functionalities according to the traffic demand [9], and demonstrated that such an adaptation mechanism allows a great energy saving especially in low traffic scenarios. The investigations motivate the researchers to enhance power savings at IoT relays/APs by applying similar methods. That is, to exploit the potential of energy saving by tracing the traffic variations and adapting the states of relays/APs accordingly. Inevitably, under such a scheme, transmitters may suffer from a disruption in their connectivity to servers (e.g., relays or APs). As such, an extra delay is incurred. Fortunately, this 
is allowable for services without real-time constraints. In practice, the delay tolerant network (DTN) scheduling has recently been recognized as an appropriate technique to balance the available resources and communication tasks without compromising the perceived Quality of the Service (QoS) [8][15]. There have been a series of research efforts on delaytolerant IoT networks [10]-[12]. In [10], the authors proposed a delay-tolerant architecture for internet of public bikes by applying reliable buffer management. Moreover, an optimized delay-tolerant approach was designed for integrated RFID IoT networks [11], while a standard-based M2M platform was configured to collect data from sensors with strict energy constraints [12]. In this paper, we focus on the intermittent transmission scheme in relay-assisted DTN networks for the energy-saving sake without violating the delay constraint.

\section{B. Related Works}

The relevant researches on IoT-relaying networks can mainly be divided into two categories: 1) network architecture and routing protocol designs [5];2) resource management, including relay number determination [13], [14], placement [15], device-relay-channel association [13]. In [13], [14], optimal relay placements and device-relay-channel associations were determined to achieve energy efficient uplink transmissions. The relay placement problem under a simplified delay constraint was investigated in [15]. Nevertheless, the above relay research works did not consider packet transmitting scheduling in time domain.

Quite recently, traffic-aware scheduling problems in conventional cellular networks have grasped considerable attentions [16]-[18]. By configuring the lightly-loaded relays into their sleeping state, the working duration of relays/APs is reduced, bringing the benefit of a lowered basic electrical consumption. In [16], an interesting aerial flying network infrastructure was envisioned, which enables the network topology to be dynamically reconfigured to match the users' traffic demands. Similar work can be found in [8], [18] and [19], where nodes sleeping and user transmission scheduling were investigated, aiming at diminishing the energy consumptions by exploiting both temporal and spatial variation of traffic loads. In addition, some important and pertinent research works on traffic-aware communication networks were performed in [20], [21], where an $N$-threshold base stations service rule has been applied to one-hop transmissions. The authors illustrated that one optimum threshold exists in terms of the energy-delay tradeoff. However, the design is infeasible in the relay-assisted transmission scheme.

Inherently, traffic-aware relay-assisted two-stage transmissions implemented by adaptively configuring the relays/APs' service states can be modelled as a two-stage queueing system. To the best of our knowledge, very few researches are performed for such network settings. The most relevant works are [22]-[25], where two-stage M/G/1 queueing systems have been studied. An $N$-threshold relay service rule was also applied. Although [22]-[25] presented significant insights for two-stage queueing networks, they investigated the scenarios where the first stage was in the batch-service mode and cannot apply to wireless relaying system. In relaying wireless communication networks, however, users are mainly served in an individual mode during each hop. Some research works on two-stage queueing networks with individual-service mode are [15] and [26]. However, they supposed a relay always-on service policy, and failed to take into account the power-saving relevant queueing service design.

The traffic-aware $N$-threshold transmission scheme inherently differentiates from the previously published literature [20]-[26]. In particular, the mean waiting time, as a key performance metric, is typically composed of the queuing delay, transmission delay as well as processing delay. Rigorous mathematical analysis for the mean waiting time along with the overall power consumption that includes the electrical consumption has not been investigated yet. The mean waiting time, in particular, cannot be simply obtained from the widely adopted Little's Law, since it is closely related with the service stage when the packet arrives. Furthermore, the expected power consumption is related to the states of the relay and AP along with the time span that they remain in those states. Hence, the power consumption model is also different from those in the published literature [20]-[26].

\section{Contributions}

We focus on seeking low-power solutions while guaranteeing the transmitters' delay performance for delay-tolerant networks. One fundamental and critical issue is to investigate how two key metrics, i.e., energy consumption and mean waiting time are related with packet arrival rate, queueing service rate, the service threshold, etc. To the best of our knowledge, relay-aided two-stage queueing communication service is still an open issue and different from the stateof-the-art. Three critical and technically challenging issues are: 1) derivation of the expected queuing length in different service stages; 2) formulation of the mean waiting time; and 3) determination of energy-saving service threshold that adapts to various mean waiting time tolerance and packet arrival rates.

Specifically, our main contributions are listed as follows:

- A more general and practical two-hop queueing system is investigated. Two random processes are considered, including the ergodic fading channels and Poisson arrival process. Additionally, the power consumption model is more practical in the sense that we take into account the electrical circuit energy consumptions when nodes are in different modes as well as power costs for mode transitions.

- As a key metric, the mean waiting time is first derived. The mean waiting time hence can be measured mathematically. Based on that, the long-term expected power consumption is formulated. Then, how they are affected by the system parameters, namely packet arrival rate, channel statistics, and relay service threshold is mathematically analysed.

- $\quad$ By adopting tight performance bounds based on Jensen's inequality, complicated manifold integral computations are avoided. This method enables our results to be derived in tractable closed-formulae, and 
evaluated quite easily. Hence, the overall computational complexity is greatly reduced.

- $\quad$ The optimum service threshold that can be flexibly adjusted to packet arrival rate is determined such that the long-term expected power consumption is minimized without violating the delay constraint.

The rest of the paper is organized as follows. In Section II, we present the system model. Queueing behaviour analysis is given in Section III. In Section IV, the considered power minimization problem is formulated and analyzed. Problem approximation and determination are provided in Section V. The analytical and simulation results are presented in Section VI. Finally, Section VII concludes this paper.

TABLE I

NOTATIONS

\begin{tabular}{|c|c|}
\hline Notation & Description \\
\hline$N_{0}$ & $\begin{array}{l}\text { the number of accumulated packets at the } \\
\text { beginning of the first service stage (FSS) }\end{array}$ \\
\hline$N_{1}$ & the number of arrivals during $N_{0}$ packets' FSS \\
\hline$N_{2}$ & $\begin{array}{l}\text { the number of accumulated packets at the end } \\
\text { of the second service stage }\end{array}$ \\
\hline$N_{0}(z), N_{1}(z), N_{2}(z)$ & PGFs of $N_{0}, N_{1}, N_{2}$ \\
\hline $\mathcal{L}_{T_{1}}(\theta), \mathcal{L}_{T_{2}}(\theta)$ & $\begin{array}{l}\text { Laplace-Stieltjes transforms (LSTs) of } T_{1}(t) \text {, } \\
T_{2}(t)\end{array}$ \\
\hline
\end{tabular}

\section{SySTEM MODEL}

As depicted in Fig. 1] we consider a two-stage and singlerelay queueing IoT communication system consisting of users, a relay station and an AP that is connected to the Internet. The users can be distributed sensors, actuators, cameras for smart farm/industrial/forestry applications. The AP can be a data integration point in the practical IoT scenarios. Besides, we assume a central-controlled network framework. The central node can be the relay or one dedicated node that is capable to communicate with each transceiver in the network. Once a packet is generated at one specific user, the user notifies the central node of its existence via a short message (e.g., beacon message). The packet arrivals at the central node follow the Poisson distribution with average rate $\lambda$ (measured in packets/ms). Note that the value of $\lambda$ varies with the statistic traffic load.

Uplink data transmissions are considered, i.e. the users intend to upload their packets to the AP. However, the direct wireless communication links between the users and AP are unavailable due to the long-distance, severe fading or physical obstacles. Additionally, no Internet connectivity is available. As such, a relay station is applied to wirelessly collect data from sensors and forward them to the AP. Specifically, a twostage service is conducted to deliver the arriving packets to the AP via the the relay. The relay is equipped with a single antenna and acts as a server in the queueing system. The AP-Internet transmissions can be realized with high-capacity wired cables. We only elaborate on the two-stage wireless transmissions, while AP-Internet transmissions are ignored in the paper.

The packet queue is managed by the central node. In what follows, a sub-cycle is first introduced, which is composed of two service stages, i.e., the first service stage (FSS) and second service stage (SSS), respectively. In the FSS, the users transmit their packets to the relays sequentially. In the SSS, the packets received at the relay are successively forwarded to the AP. We take an arbitrary sub-cycle below as an example to illustrate the service model. Further, the definition of a regeneration cycle is introduced, which consists of several consecutive subcycles. Note that the notations are listed in Table I]

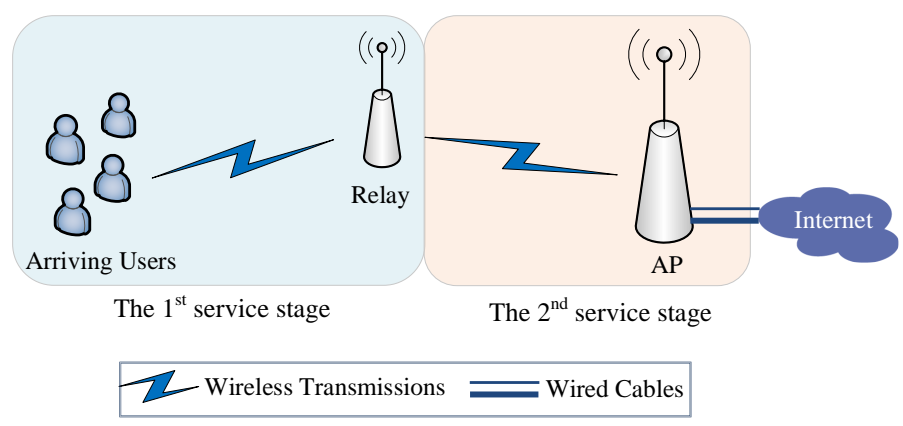

Fig. 1. Two-stage queueing service model.

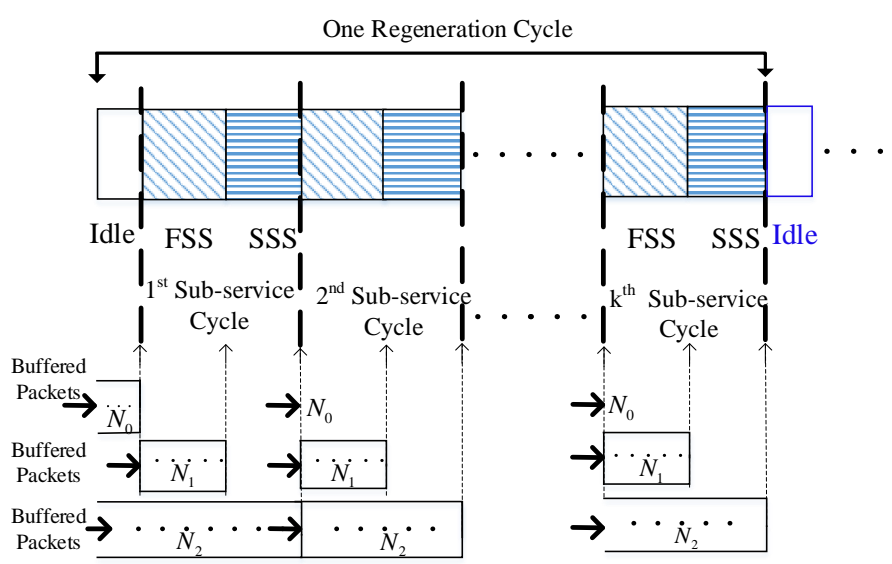

Fig. 2. One Regeneration Cycle Model Illustration.

\section{A. The First Service Stage (FSS)}

The $N$-threshold policy is applied. The detailed policy is illustrated as follows. Let $N_{2}$ represent the number of accumulated packets at the end of the second service stage (which will be illustrated in Subsection II-B). Only when $N_{2}>0$, the relay server switches immediately into the receiving mode at the end of the SSS1 In case that $N_{2}=0$, the relay server remains in the listening mode till the number of newly arrived packets reaches the predefined threshold $N$, i.e.,

$$
N_{0}= \begin{cases}N_{2}, & \text { if } N_{2}>0, \\ N, & \text { otherwise. }\end{cases}
$$

Based on our definition of $N_{0}$, we can observe that $N_{0}$ is inherently a random variable, since $N_{2}$ brings randomness

\footnotetext{
${ }^{1}$ When the number of accumulated packets satisfies a pre-set rule, the relay sends the packet-transmitting-request to the waiting users. After that, the relay immediately switches into the receiving mode.
} 
to it. Besides, note that during the FSS, the AP remains listening. Specifically, in the listening mode, a transceiver is active and ready but not currently receiving or transmitting packets. Listening to the channel and executing clear channels assessment are the main activity in the listening mode 2

Additionally, a gated-policy is adopted at the relay. Specifically, only the packets that are already present (before the FSS starts) will be served in the current sub-cycle, while those arrive during the current FSS have to wait for being served in the next sub-cycle.

In the MAC layer, a schedule-based MAC protocol, i.e., time division multiple access (TDMA) is applied to coordinate packet transmitting among the users. Specifically, the packets are individually served by the relay server in the first come first served (FCFS) rule. Note that packet collision is avoided under the TDMA-based MAC protocol. In the FSS, the active relay keeps receiving packets, decoding and storing them in its data buffer. It is assumed that the buffer size is large enough to store the packets, which is reasonable since in practice, only a limited number of packets are accumulated in a sub-cycle; otherwise, the waiting time will be intolerable.

In the FSS, the service rate is

$$
C_{1}\left(h_{1}\right)=B \log \left(1+\frac{\left|h_{1}\right|^{2} p_{1}}{\sigma_{1}^{2}}\right),
$$

where $B$ is the bandwidth; $p_{1}$ is the data transmitting power of the packets; $\sigma_{1}^{2}$ is the noise power; $h_{1} \sim \mathcal{C N}\left(0, \sigma_{h_{1}}^{2}\right)$ is the channel gain; $\mathcal{C N}(m, v)$ denotes a complex Gaussian random variable with $m$ and $v$ standing for the mean value and variance, respectively; $\left|h_{1}\right|$ is the amplitude of $h_{1}$ and follows the Rayleigh distribution; $\sigma_{h_{1}}^{2}$ is the variance of Rayleigh distribution. Rayleigh fading channels are assumed to be independent and identically distributed (i.i.d.) for mathematical tractability and drawing insights.

The $i$ th arriving packet is indexed by packet $i$. The service time span for individual packet (or individual service time span for short), denoted by $\left\{T_{1, i}, i=1,2 \cdots,\right\}$, in the FSS are assumed to be a stationary i.i.d. process with a distribution function, $T_{1}(t)$. Note that such an assumption can be realizable in practice. One feasible way is to flexibly match each packet's transmitting power with the channel stochastic parameters, such that the service rate is a stationary i.i.d. process. It is further assumed that the length of each individual packet is fixed and denoted as 3 The individual service time is obtained as

$$
T_{1}=l / C_{1}\left(h_{1}\right),
$$

while its distribution function, denoted as $T_{1}(t)$, is

$$
T_{1}(t)=\operatorname{Pr}\left\{\frac{l}{C_{1}\left(h_{1}\right)}<t\right\} .
$$

\footnotetext{
${ }^{2}$ The AP and relay is not completely shut off because it may be quite challenging to entirely turn off the AP and then require it switch to the receiving mode immediately for the next second service stage. Nevertheless, listening mode control within a short time span is feasible, as investigated in [28].

${ }^{3}$ If the length is not fixed, we can split longer packets into shorter ones such that the length of each packet is the same.
}

Further, by combining with (2), $T_{1}(t)$ can be presented as follows:

$$
T_{1}(t)=\exp \left(-\frac{\left(\exp \left(\frac{l}{B t}\right)-1\right) \sigma_{1}^{2}}{\sigma_{h_{1}}^{2} p_{1}}\right) .
$$

\section{B. The Second Service Stage (SSS)}

Once the FSS is completed, the SSS starts and the AP switches from the listening mode to the receiving mode. All the packets stored in the relay's buffer will be forwarded to the AP according to the FCFS rule. The individual service time span in the SSS, denoted by $\left\{T_{2, i}, i=1,2 \cdots,\right\}$, is assumed to be a stationary i.i.d. process with a distribution function $T_{2}(t)$. Note that new arrivals during the SSS will wait for the next "FSS+SSS" sub-cycle service.

The service rate in the SSS, denoted as $C_{2}\left(h_{2}\right)$, can be given by

$$
C_{2}\left(h_{2}\right)=B \log \left(1+\frac{\left|h_{2}\right|^{2} p_{2}}{\sigma_{2}^{2}}\right),
$$

where $p_{2}$ is the data transmitting power at the relay; $h_{2}$ and $\sigma_{2}^{2}$ are defined respectively similar to $h_{1}$ and $\sigma_{1}^{2}$, and correspond to the parameters for the relay-AP channel in the $\mathrm{SSS}_{4}^{4}$.

Correspondingly, the individual service time in the SSS can be obtained as

$$
T_{2}=l / C_{2}\left(h_{2}\right) .
$$

$\left\{T_{2, i}, i=1,2 \cdots,\right\}$ is assumed to be a stationary i.i.d. process with a distribution function $T_{2}(t)$. The detailed expression for $T_{2}(t)$ can be obtained by changing " 1 " in (5) into " 2 ". We omit its expression here.

\section{The Regeneration Cycle Model}

It is noteworthy that intermittent wireless transmission scheme is applied. Specifically, at the end of the SSS, depending on the value of $N_{2}$, the relay server decides whether it starts the next sub-service cycle immediately or not. As described in Section II-A if $N_{2} \geq 0$, the next sub-service cycle will start, resulting in consecutive sub-service cycles; otherwise, the sub-service cycle will be followed by an idle period.

As depicted in Fig. 2, a regeneration cycle is introduced and defined as the serving process between two idle states, consisting of an idle period followed by several consecutive sub-service cycles. The number of sub-service cycle is a random variable, denoted as $k$. Statistically, $k$ also represents the number of sub-cycle services before the service turns into idle mode, indicating that $k$ follows the geometric distribution. Hence, we have

$$
\mathbb{E}\{k\}=1 / \pi_{0},
$$

where $\mathbb{E}\{\cdot\}$ is the expectation operator; $\pi_{0}=\operatorname{Pr}\left\{N_{2}=0\right\}$, and equals to the probability that the relay switches from the transmitting mode to the listening mode.

For the above queueing service model, we will analyse its key measures in the sequel, including the regeneration cycle time span, queue lengths at different service stages, based on which the mean waiting time is gradually derived.

\footnotetext{
${ }^{4} T_{2}$ and $T_{2}(t)$ can be directly obtained by substituting index "1" in 3 and (4) with " 2 ". The details are omitted here.
} 


\section{Regeneration Cycle Measures Analysis}

\section{A. The Regeneration Cycle Time Span}

Denote the number of packet arrivals in one complete regeneration cycle as $\Gamma . \mathbb{E}\{\Gamma\}$ is the expected number of arrived packets in one complete regeneration cycle. In what follows, we respectively calculate the expected number of packet arrivals during the idle, FSSs and SSSs.

First, note that in the idle mode, the relaying service will not start until there are $N$ packets waiting in the queue. Additionally, there are in total $\Gamma$ arrived packets, each taking an average of $\mathbb{E}\left\{T_{1}\right\}$ and $\mathbb{E}\left\{T_{2}\right\}$ seconds for the FSS and SSS, respectively. According to Wald's equation [23], it takes an average of $\mathbb{E}\{\Gamma\} \mathbb{E}\left\{T_{1}\right\}$ and $\mathbb{E}\{\Gamma\} \mathbb{E}\left\{T_{2}\right\}$ seconds for serving an average of $\mathbb{E}\{\Gamma\}$ packets in the FSSs and SSSs of one complete regeneration cycle, respectively. Based on the delay cycle property in Section 1.2 in [23], we achieve that the total expected numbers of packet arrivals in the idle stage, FSSs, and SSSs are $N, \lambda \cdot \mathbb{E}\{\Gamma\} \mathbb{E}\left\{T_{1}\right\}$ and $\lambda \cdot \mathbb{E}\{\Gamma\} \mathbb{E}\left\{T_{2}\right\}$, respectively (for detailed derivations, please refer to Appendix VIII-A). $\mathbb{E}\{\Gamma\}$ can be given by

$$
\mathbb{E}\{\Gamma\}=N+\lambda \cdot \mathbb{E}\{\Gamma\} \mathbb{E}\left\{T_{1}\right\}+\lambda \cdot \mathbb{E}\{\Gamma\} \mathbb{E}\left\{T_{2}\right\} .
$$

Let $\mathbb{E}\left\{T_{0}\right\}$ represent the expected idle duration. Since the packet arrival follows the Poisson distribution, $\mathbb{E}\left\{T_{0}\right\}$ is related with $N$ as follows:

$$
\mathbb{E}\left\{T_{0}\right\}=\frac{N}{\lambda} .
$$

From (9), we obtain that the closed-form of $\mathbb{E}\{\Gamma\}$ can be expressed as

$$
\mathbb{E}\{\Gamma\}=\frac{N}{1-\lambda \mathbb{E}\left\{T_{1}\right\}-\lambda \mathbb{E}\left\{T_{2}\right\}} .
$$

Let us denote $X_{i}=\left|h_{i}\right|^{2}, Y_{i}=\frac{l}{C_{i}\left(X_{i}\right)}(i=1,2)$, for convenience. Since $X_{i}=\left|h_{i}\right|^{2}$ follows an exponential p.d.f. [23], such that

$$
g_{X_{i}}(x)=\frac{1}{\sigma_{h_{i}}^{2}} \exp \left(-\frac{x}{\sigma_{h_{i}}^{2}}\right) .
$$

Further, $\mathbb{E}\left\{T_{i}\right\}(i \in\{1,2\})$ in 9 and 11 is calculated as

$$
\mathbb{E}\left\{T_{i}\right\}=\int_{0}^{+\infty} \frac{l}{C_{i}(x)} g_{X_{i}}(x) d x{ }^{5}
$$

Let $T_{R C}$ represent the time span of one regeneration cycle. $\mathbb{E}\left\{T_{R C}\right\}$ can be derived as (for detailed derivations, please refer to Appendix VIII-A

$$
\mathbb{E}\left\{T_{R C}\right\}=\frac{\mathbb{E}\{\Gamma\}}{\lambda} .
$$

Direct derivations of the mean waiting time are considerably challenging. Note that the mean waiting time can be obtained by taking the derivatives of the LST of the distribution of the waiting time. The LST of the distribution of the waiting time can be achieved from the probability generating functions (PGFs) of the queue length.

\footnotetext{
${ }^{5}$ Note that in practice, $X_{i}=\left|h_{i}\right|^{2}>0$. For mathematical computation, we bound $x$ as $x \geq \delta$, where $\delta$ takes a considerably small value, e.g., $10^{-6}$.
}

In what follows, we will respectively formulate the PGFs of the FSS and SSS queue lengths, based on which the mean waiting time is further deduced.

\section{B. PGFs of the FSS and SSS Queue Lengths}

Let $N_{1}$ represent the number of arrivals during $N_{0}$ packets' FSS, and $N_{0}$ represent the number of accumulated packets at the beginning of the first service stage (FSS). Let $N_{0}(z)$, $N_{1}(z), N_{2}(z)$ represent the PGFs of $N_{0}, N_{1}, N_{2}$. In the following, the deductions of $N_{0}(z), N_{1}(z)$ and $N_{2}(z)$ are illustrated first. On this basis, $\pi_{0}$ is derived.

According to the relations between the LST and PGF of the Poisson arrival process (interested readers are referred to Page 5 of [23]), $N_{0}(z), N_{1}(z)$ and $N_{2}(z)$ can be achieved from the LST of the distribution function $T_{i}(t), i=1,2$. In this subsection, we first derive the LSTs.

Let us denote $Y_{i}=\frac{l}{C_{i}\left(X_{i}\right)}(i=1,2)$, for convenience. Let $g_{Y_{i}}(y)$ represent the probability density function (p.d.f.) of $Y_{i}$. By definition of LST (for interested readers, please refer to Eq. (1.4a) in [23]), we obtain the LST of the distribution function $T_{i}(t)$ as

$$
\mathcal{L}_{T_{i}}(\theta)=\int_{0}^{+\infty} \exp (-\theta y) g_{Y_{i}}(y) d y \text {. }
$$

$Y_{i}=\frac{l}{C_{i}\left(X_{i}\right)}$ monotonically decreases with $X_{i}$, then $g_{Y_{i}}(y)$ is obtained as $g_{Y_{i}}(y)=\frac{g_{X_{i}}(x)}{\left|\nabla_{x} y\right|}$ [29], where $\nabla_{x} y$ is the derivative of $y$ w.r.t. $x$. Finally, 14 can be rewritten as

$$
\mathcal{L}_{T_{i}}(\theta)=\int_{0}^{+\infty} \exp \left(-\theta \frac{l}{C_{i}(x)}\right) g_{X_{i}}(x) d x .
$$

Note that the PGFs of the number of packet arrivals during one packet's first and second service stage can be respectively calculated as $\mathcal{L}_{T_{1}}(\lambda-\lambda z)$ and $\mathcal{L}_{T_{2}}(\lambda-\lambda z)$ (see page 5 of [23])). Then, during the overall individual service time of $N_{0}$ accumulated packets, the PGFs of the number of packet arrivals can be given as

$$
N_{1}(z)=\sum_{i=0}^{+\infty} \operatorname{Pr}\left\{N_{0}=i\right\}\left\{\mathcal{L}_{T_{1}}(\lambda-\lambda z)\right\}^{i} .
$$

According to the definition of PDF, we rewrite $N_{1}(z)$ as

$$
N_{1}(z)=N_{0}\left(\mathcal{L}_{T_{1}}(\lambda-\lambda z)\right)
$$

Note that (17) also reveals the relationship between $N_{1}(z)$ and $N_{0}(z)$.

Further, by definitions of $N_{2}$ and $N_{0}$, the relations between $N_{2}(z)$ and $N_{0}(z)$ are

$$
\begin{aligned}
& N_{2}(z)=N_{0}\left(\mathcal{L}_{T_{1}}(\lambda-\lambda z)\right) \cdot N_{0}\left(\mathcal{L}_{T_{2}}(\lambda-\lambda z)\right), \\
& N_{0}(z)=N_{2}(z)-\pi_{0}+\pi_{0} z^{N} .
\end{aligned}
$$

It can be noticed from (17)-(19) that $N_{0}(z)$ is the kernel of the derivations on $N_{1}(z)$ and $N_{2}(z)$. For $N_{0}(z)$, we have the following Proposition: 
Proposition 1. For the two-stage service, where the individual service time span is i.i.d., $N_{0}(z)$ can be given as

$$
\begin{aligned}
N_{0}(z)= & \left(\left[\left(1-g_{n-1}(z)\right)^{2}-g_{n-2}(z)\right]^{2}-\cdots-g_{1}(z)\right)^{2} \\
& -g_{0}(z),
\end{aligned}
$$

where $n \in \mathbb{N}^{+}$and $n \rightarrow \infty ; g_{n}(z)(\forall n)$ is defined in (62).

Additionally, $N_{0}(z)$ absolutely converges for any $|z|<1$. $N_{1}(z)$ and $N_{2}(z)$ can be further obtained from (17) and (18). Their detailed expressions are omitted here.

Proof. The proof is presented in Appendix VIII-B

Proposition 1 provides a general PGF formula. The derivations in 62)-(66) indicate that $N_{0}(z)$ is a function w.r.t. $N$, $\pi_{0}$, and the queueing service parameters in (15), including $l$, $B$, and $\sigma_{h_{i}}^{2}(i=1,2)$.

In particular, Proposition 1 forms a fundamental basis for all subsequent derivations and provides two following clues. On the one hand, the PGF property that $N_{0}(0)=\operatorname{Pr}\left\{N_{0}=0\right\}$ provides a clue to investigate the relationship among different parameters, e.g., $N, \pi_{0}$ and the queueing service parameters in (15). On the other hand, $N_{0}(z)$ can be directly obtained once $z$ is given, which is useful in deriving the LST of different service stages. In particular, by replacing $\mathcal{L}_{T_{i}}(\theta)$ with $z$, we can easily obtain the LST of the distribution function of $N_{0}$ packets' service time span (either in the FSS or the SSS, or a smaller-scale stage). That is, $N_{0}(z)$ also provides clues in deriving the mean service duration.

As can be found in Proposition 1, $\pi_{0}$ is a fundamental and critical parameter in obtaining $N_{0}(z)$ in $(20)$ as well as subsequent derivation of the mean waiting time. On the other hand, according to $8, \pi_{0}$ determines the mode transmission frequency at the AP. It is closely related to power consumption at the AP. For $\pi_{0}$, Proposition 2 is provided as follows:

Proposition 2. For the two-stage queueing service, where the individual service time span is i.i.d., $\pi_{0}$ is

$$
\pi_{0}=\left\{N_{0}\left(\mathcal{L}_{T_{2}}(\lambda)\right)\right\}^{2} .
$$

Proof. The proof is relegated in Appendix VIII-C

Proposition 2 sheds light on the relationship between $\pi_{0}$ and various system parameters, including the packet arrival rate, channel statistics, and relay service threshold. Although (21) is not in an explicit-form, $\pi_{0}$ can be numerically determined efficiently with the approximation method that will be illustrated in Section V.

\section{Mean Waiting Time}

Throughout the paper, the waiting time represents the duration from one packet joins the queue system until its turn to be served in the SSS, which is composed of the queuing delay, processing delay as well as transmission delay. Their specific meanings are presented as follows.

The queuing delay represents the time span from the instant a packet is generated (equivalent to the instant when the packet enters the queue) till the instant it starts to be transmitted. Clearly, it is dependent on the traffic intensity as well as the link service rate. The processing delay is the time duration spent for (de)coding, (de)modulation, etc, which is ignorable. On the other hand, under the assumption that the CSI is known at the transmitters and with a maximum rate of the Shannon capacity, the packet can be correctly received and retransmissions are avoided, theoretically. Each packet's transmission delay can be obtained from (3) and (7).

In this section, the queuing delay as well as transmission delay are derived. One observed packet has to wait for its data being forwarded to the AP in the following three cases:

- Case 1: the observed packet arrives when the server is idle: In case 1 , the observed packet, denoted by $u_{i^{\prime}}(i \in$ $\{1,2, \cdots, N\}$ ) will wait

(i) the remaining idle period, plus

(ii) the time span of the FSS, plus

(iii) the overall SSS time span of packets that arrive prior to $u_{i^{\prime}}$.

$$
\begin{aligned}
W_{q}(\theta \mid \text { case } 1)= & \sum_{i=1}^{N} \operatorname{Pr}\left\{i^{\prime}=i\right\} \cdot\left\{\mathcal{L}_{T_{I N T}}(\theta)\right\}^{N-i} \\
& \cdot\left\{\mathcal{L}_{T_{1}}(\theta)\right\}^{N}\left\{\mathcal{L}_{T_{2}}(\theta)\right\}^{i-1},
\end{aligned}
$$

where $\operatorname{Pr}\left\{i^{\prime}=i\right\}$ represents the probability that the observed packet ranks the $i$ th among $N$ packets with respect to (w.r.t.) the arrival time, and can be calculated as $\operatorname{Pr}\left\{i^{\prime}=i\right\}=\frac{1}{N}$; $\mathcal{L}_{T_{I N T}}(\theta)$ is LST of the distribution function of the time interval between any two neighbouring arrivals. For Poisson arrivals, $\mathcal{L}_{T_{I N T}}(\theta)$ can be computed as follows:

$$
\mathcal{L}_{T_{I N T}}(\theta)=\int_{0}^{+\infty} \exp (-\theta x)(\lambda \exp (-\lambda x)) d x=\frac{\lambda}{\theta+\lambda} .
$$

- Case 2: The observed packet arrives during the FSS:

Let $T^{\prime}$ represent the total individual service time span of $N_{0}$ packets in the FSS. The LST of the distribution function of $T^{\prime}$ can be expressed as

$$
\mathcal{L}_{T^{\prime}}(\theta)=N_{0}\left(\mathcal{L}_{T_{1}}(\theta)\right) .
$$

One observed packet arrives during the FSS will wait

(i) the remaining time of $T^{\prime}$ period, plus

(ii) the overall SSS time span of $N_{0}$ packets in the currently undergoing sub-service cycle, plus

(iii) the overall FSS time span of $N_{0}$ packets in the next sub-service cycle, plus

(iv) the overall SSS time span (in the next sub-service cycle) of the packets that arrive during the elapsed time of $T^{\prime}$ period.

Note that (i)+(iv) can be obtained according to the joint LST property of the elapsed and remaining service time distribution function, i.e.,

$$
X_{ \pm}^{(\text {case } 2)}(\theta)=\frac{\mathcal{L}_{T^{\prime}}\left(\lambda-\lambda \mathcal{L}_{T_{2}}(\theta)\right)-\mathcal{L}_{T^{\prime}}(\theta)}{\left(\theta-\lambda+\lambda \mathcal{L}_{T_{2}}(\theta)\right) \mathbb{E}\left\{T^{\prime}\right\}} .
$$

For detailed derivations, interested readers can refer to Eq.(1.54b) in [23]. Further, according to the property of the PGF, namely, PGF of sums of independent random variables 
equals to the multiplication of PGF of each independent random variable (which is referred to as the Sum-Multiplication property in the following), the LST of the distribution function of waiting time in Case 2 is finally given as

$$
W_{q}(\theta \mid \text { case } 2)=X_{ \pm}^{(\text {case } 2)}(\theta) \cdot N_{0}\left(\mathcal{L}_{T_{2}}(\theta)\right) \cdot N_{0}\left(\mathcal{L}_{T_{1}}(\theta)\right)
$$

where $\mathbb{E}\left\{T^{\prime}\right\}$ can be obtained by differentiating (24) w.r.t. $\theta$. Specifically, we have

$$
\mathbb{E}\left\{T^{\prime}\right\}=-\left.\nabla_{\theta} \mathcal{L}_{T^{\prime}}(\theta)\right|_{\theta=0} .
$$

- Case 3: the observed packet joins the waiting queue during the SSS: Let $T^{\prime \prime}$ denote the total SSS time span of $N_{0}$ packets. The LST of the distribution function of $T^{\prime \prime}$ is represented as

$$
\mathcal{L}_{T^{\prime \prime}}(\theta)=N_{0}\left(\mathcal{L}_{T_{2}}(\theta)\right)
$$

One observed packet arrives during the SSS will wait

(i) the remaining time of $T^{\prime \prime}$ period, plus

(ii) the overall FSS time span of $N_{0}$ packets in the next sub-service cycle, plus

(iii) the overall SSS time span of packets that arrive during the FSS of the currently undergoing subservice cycle, plus

(iv) the overall SSS time span of the packets that arrive during the elapsed time of $T^{\prime \prime}$ period.

Similar to 25, (i)+(iv) can be obtained according to the joint LST property of the elapsed and remaining service time distribution function, which can be expressed as

$$
X_{ \pm}^{\left(\text {case }^{\text {s })}\right.}(\theta)=\frac{\mathcal{L}_{T^{\prime \prime}}\left(\lambda-\lambda \mathcal{L}_{T_{2}}(\theta)\right)-\mathcal{L}_{T^{\prime \prime}}(\theta)}{\left(\theta-\lambda+\lambda \mathcal{L}_{T_{2}}(\theta)\right) \mathbb{E}\left\{T^{\prime \prime}\right\}} .
$$

The LST of the distribution function of the waiting time in this case can be calculated as

$$
\begin{aligned}
W_{q}(\theta \mid \text { case } 3)(\theta)= & X_{ \pm}^{(\text {case } 3)}(\theta) \cdot N_{0}\left(\mathcal{L}_{T_{1}}(\theta)\right) . \\
& N_{0}\left(\mathcal{L}_{T_{1}}\left(\lambda-\lambda \mathcal{L}_{T_{2}}(\theta)\right)\right),
\end{aligned}
$$

where, similar to the expression of $\mathbb{E}\left\{T^{\prime}\right\}, \mathbb{E}\left\{T^{\prime \prime}\right\}$ can be illustrated as

$$
\mathbb{E}\left\{T^{\prime \prime}\right\}=-\left.\nabla_{\theta} \mathcal{L}_{T^{\prime \prime}}(\theta)\right|_{\theta=0} .
$$

Poisson arrivals see time averages (PASTA) (i.e., the fraction of arrivals that see the process in some state is equal to the fraction of time span when the process is in that state) holds for our two-phase M/G/1 queueing system [30], the probabilities that cases 1, 2 and 3 occur can be formulated as

$$
\begin{aligned}
& \operatorname{Pr}\{\text { Case } 1\}=\frac{\mathbb{E}\left\{T_{0}\right\}}{\mathbb{E}\left\{T_{R C}\right\}}=1-\lambda \mathbb{E}\left\{T_{1}\right\}-\lambda \mathbb{E}\left\{T_{2}\right\}, \\
& \operatorname{Pr}\{\text { Case } 2\}=\frac{\mathbb{E}\{\Gamma\} \mathbb{E}\left\{T_{1}\right\}}{\mathbb{E}\left\{T_{R C}\right\}}=\lambda \mathbb{E}\left\{T_{1}\right\}, \\
& \operatorname{Pr}\{\text { Case }\}=\frac{\mathbb{E}\{\Gamma\} \mathbb{E}\left\{T_{2}\right\}}{\mathbb{E}\left\{T_{R C}\right\}}=\lambda \mathbb{E}\left\{T_{2}\right\},
\end{aligned}
$$

where $\mathbb{E}\left\{T_{0}\right\}$ is defined in 10 .
To keep the stability of the two-stage queueing system, the busy probability of the relay server must be less than 1; namely, $\operatorname{Pr}\{$ Case 2$\}+\operatorname{Pr}\{$ Case 3$\}<1$. Equivalently, it is required that

$$
\lambda<\frac{1}{\mathbb{E}\left\{T_{1}\right\}+\mathbb{E}\left\{T_{2}\right\}} .
$$

must be satisfied.

Finally, the following proposition can be presented.

Proposition 3. In a two-stage queueing system with i.i.d. individual service time span, the mean waiting time of one packet is

$$
\begin{aligned}
\mathbb{E}\left\{W_{q}\right\}=- & \left.\nabla_{\theta}\left(W_{q}(\theta \mid \text { case } 1) \cdot \operatorname{Pr}\{\text { Case } 1\}\right)\right|_{\theta=0} \\
& -\left.\nabla_{\theta}\left(W_{q}(\theta \mid \text { case } 2) \cdot \operatorname{Pr}\{\text { Case } 2\}\right)\right|_{\theta=0} \\
& \left.-\nabla_{\theta}\left(W_{q}(\theta \mid \text { case } 3) \cdot \operatorname{Pr}\{\text { Case } 3\}\right)\right)\left.\right|_{\theta=0} .
\end{aligned}
$$

Proof. The proof can be found in Appendix VIII-D

Proposition 3 provides a general formula for the mean waiting time, which relates the mean waiting time with multiple queueing system parameters, namely packet arrival rate, channel statistics, and relay service threshold. It is more computationally efficient than Monte-Carlo simulations and provides guidelines for engineering design.

\section{Power Minimization Problem Formulation}

Based on the prior analytic packet delay result, in this section we further illustrate the power minimization problem. Before that, the long-term average power consumption at different service stages has to be first formulated.

\section{A. Power Consumption Formulation}

A more general and practical power consumption model is considered. Specifically, the overall power consumed by the network is composed of three parts: energy consumed at the packets, relays, and AP. The consumed energy supports: i) mode transitions between the listening and receiving modes, ii) packets receiving in the FSS, iii) packets transmitting at the relay and receiving at AP (both in the SSS).

Based on our model, each transceiver is in listening, transmitting, or receiving mode. Specifically, the transceivers' states in the idle, FSS, and SSS are listed in Table II.

TABLE II

TRANSCEIVERS' STATES

\begin{tabular}{c||c|c|c}
\hline Stage & Users' state & Relay's state & AP's state \\
\hline \hline$I d l e$ & listening & listening & listening \\
\hline$F S S$ & listening/transmitting & receiving & listening \\
\hline$S S S$ & listening & transmitting & receiving \\
\hline
\end{tabular}

In the sequel, we formulate the expected energy consumption during one regeneration cycle, based on which the average power consumption is formulated. 
1) Power Consumption at Users: The power consumption in waiting and transmitting modes is [32]:

$$
P_{u}= \begin{cases}P_{0, u}, & \text { waiting mode, } \\ P_{0, u}+\triangle_{P_{u}} p_{1} \triangleq P_{T, u}, & \text { transmitting mode }\end{cases}
$$

where $P_{0, u}$ represents the basic electrical circuit power consumption; $\triangle_{P_{u}}$ is the slope of the load-dependent power consumption. Note that the waiting users are not shut off when they are in the waiting mode, since they have to listen to the packet-transmitting-request from the relay, as illustrated in Footnote 1.

As depicted in Fig. 2, the expected overall energy cost at users in each regeneration cycle contains energy consumed in the idle mode (with an average duration of $\mathbb{E}\left\{T_{u}^{i d l e}\right\}$ ) and transmitting mode (with an average duration of $\mathbb{E}\left\{T_{u}^{t}\right\}$ ). $\mathbb{E}\left\{T_{u}^{i d l e}\right\}$ and $\mathbb{E}\left\{T_{u}^{t}\right\}$ can be respectively formulated as

$$
\begin{aligned}
\mathbb{E}\left\{T_{u}^{i d l e}\right\} & =\mathbb{E}\left\{W_{q}\right\}-\mathbb{E}\left\{T_{1}\right\}, \\
\mathbb{E}\left\{T_{u}^{t}\right\} & =\mathbb{E}\left\{T_{1}\right\} .
\end{aligned}
$$

Hence, the expected overall energy cost at the users, denoted by $\mathbb{E}_{u}^{t o t}$, can be given as

$$
\begin{aligned}
& \mathbb{E}_{u}^{\text {tot }}=\underbrace{\mathbb{E}\{\Gamma\} \mathbb{E}\left\{T_{u}^{i d l e}\right\} \cdot P_{0, u}}_{\text {Energy cost in the idle mode }}+ \\
& \underbrace{\mathbb{E}\{\Gamma\} \mathbb{E}\left\{T_{u}^{t}\right\} \cdot P_{T, u}}_{\text {Energy cost for data transmitting }} .
\end{aligned}
$$

2) Power Consumption at the Relay Server: For relays, the power consumption model in listening, receiving, and transmitting modes is [32]

$$
P_{R}= \begin{cases}P_{0, R}, & \text { receiving mode, } \\ P_{0, R}+\triangle_{P, R} \cdot p_{2} \triangleq P_{T, R}, & \text { transmitting mode } \\ P_{\text {listen }, R}, & \text { listening mode }\end{cases}
$$

where $\triangle_{P_{R}}$ is the slope of the load-dependent power consumption. Generally, we have $P_{0, R}>P_{\text {sleep }, R}$.

For each regeneration cycle depicted in Fig. 2, the total expected energy cost at the relay server, denoted by $\mathbb{E}_{R}^{t o t}$, includes energy consumed in the idle period (when relays are in the listening mode), $k$ FSSs (when relays are in the receiving mode) and $k$ SSSs (when relays are in the transmitting mode). Hence, $\mathbb{E}_{R}^{t o t}$ is represented by

$$
\begin{aligned}
\mathbb{E}_{R}^{\text {tot }}= & \underbrace{\mathbb{E}\left\{T_{0}\right\} \cdot P_{\text {listen, }}}_{\text {Energy cost for listening }}+\underbrace{\mathbb{E}\{\Gamma\} \mathbb{E}\left\{T_{1}\right\} \cdot P_{0, R}}_{\text {Energy cost for data receiving }}+ \\
& \underbrace{\mathbb{E}\{\Gamma\} \mathbb{E}\left\{T_{2}\right\} \cdot P_{T, R}}_{\text {Energy cost for transmitting }} .
\end{aligned}
$$

3) Power Consumption at the AP: The power consumption at the AP in listening and receiving modes is described as follows [32]:

$$
P_{A P}= \begin{cases}P_{0, A P}, & \text { receiving mode, } \\ P_{\text {listen }, A P}, & \text { listening mode, }\end{cases}
$$

Generally, we have $P_{0, A P}>P_{\text {sleep, } A P}$.
For each regeneration cycle, the listening mode duration at the AP consists of the idle period and FSSs for $\Gamma$ packets. The total expected energy cost at the relay server is

$$
\begin{gathered}
\mathbb{E}_{A P}^{\text {tot }}=\underbrace{\left(\mathbb{E}\left\{T_{0}\right\}+\mathbb{E}\{\Gamma\} \mathbb{E}\left\{T_{1}\right\}\right) \cdot P_{\text {sleep }, A P}}_{\text {Energy cost for listening }}+ \\
\underbrace{\mathbb{E}\{\Gamma\} \mathbb{E}\left\{T_{2}\right\} \cdot P_{0, A P}}_{\text {Energy cost for data receiving }} .
\end{gathered}
$$

4) Power Consumptions for Mode Transitions: Mode transitions happen at the relay when i) the service turns into the FSS from the idle mode, and ii) the service turns into the idle mode from the SSS. Additionally, the mode transition happens at the AP when i) the service turns into the SSS at the completion of the FSS, and ii) when the service turns into the idle/FSS mode at the completion of the SSS. The switching energy cost is fixed and denoted by $E_{s w}$ for each mode transition. For every individual regeneration cycle, the total mode transition energy cost is

$$
\mathbb{E}_{s w}^{t o t}=\mathbb{E}\left\{2 E_{s w}+2 k E_{s w}\right\} \stackrel{\left(c^{\prime}\right)}{=} 2 E_{s w}\left(1+\frac{1}{\pi_{0}}\right),
$$

where $2 E_{s w}$ is the energy cost for mode switching at the relay; $2 k E_{s w}$ is the energy cost for mode switching at the AP; $\left(c^{\prime}\right)$ satisfies with (8).

It is worth noting that the electrical circuit power at the relay server and AP are much higher than the packet transmitting power (e.g., 10 watts versus 0.1 watts or abound) [32]. Hence, the packet transmitting power $p_{2}$ can be ignored.

\section{B. Average Power Minimization Problem}

The power minimization problem under the queueing stability can be formulated as follows:

$$
\begin{array}{ll}
\mathbf{P 1}: & \min _{N} \mathbb{E}\left\{P_{\text {tot }}\right\}=\frac{\mathbb{E}_{u}^{t o t}+\mathbb{E}_{R}^{t o t}+\mathbb{E}_{A P}^{t o t}+\mathbb{E}_{s w}^{t o t}}{\mathbb{E}\left\{T_{R C}\right\}} \\
\text { s.t. } & \mathbb{E}\left\{W_{q}\right\} \leq D_{0},
\end{array}
$$

where $D_{0}$ in 47 is the maximum tolerable mean waiting time.

As can be seen from $(20)$, the resulting expression of $N_{0}(z)$ is in a recursive form. It is mathematically intractable to obtain the explicit formulae of the analytical solutions for $N$ and $\pi_{0}$ due to the following reasons. Firstly, it is considerably challenging to determine $n_{0} \in \mathbb{N}\left(n_{0} \leq n\right)$ at which $N_{0}(z)$ starts converging. Secondly, the polynomial terms in $N_{0}(z)$ contains $\pi_{0}$ of different orders. As implied in 20, the highest order of $\pi_{0}$ when $N_{0}(z)$ converges is $2^{n_{0}-1}$. Our numerical results in Section VI-A reveal that $n_{0}$ can be larger than 6 . The Abel Theorem [33] reveals that explicit solution does not exist for irreducible algebraic equations with the highest order being larger than 5 . Thirdly, $\pi_{0}$ is closely coupled with $N$, as implied in 200-63. The non-existence of the explicit form of $\pi_{0}$ determines that it is hard to achieve the closed-form of the global optimal $N$.

Fortunately, as illustrated in Proposition 1, $N_{0}(z)$ converges for any $|z|<1$. It motivates us to determine the solutions numerically and with a low-level complexity. The key formulations to obtain the desired metrics, including $\mathbb{E}\left\{P_{\text {tot }}\right\}$ in 
(46) and $\mathbb{E}\left\{W_{q}\right\}$ in (47) are: 1) the expectations of service time span $T_{1}$ and $T_{2}$ in (12); and 2) the expectation of LST in (15). It is really complicated to evaluate analytically their expressions. To obtain the desired metrics, manifold numerical integrations are performed, particular in 26 and (30). Straightforward numerical computations will lead to an overall high computational complexity.

The lower bound of the complexity, denoted by $C_{\text {total }}$, is

$$
\begin{aligned}
C_{\text {total }}= & \mathcal{O}\left(C_{\mathbb{E}_{T}}+\frac{n(n+1)}{2} C_{\mathcal{L}_{T}}+n(n+1) C_{\mathcal{L}_{T}}+\frac{n(n+1)}{2} C_{\mathcal{L}_{T}}\right) \\
& =\mathcal{O}\left(C_{\mathbb{E}_{T}}+2 n(n+1) C_{\mathcal{L}_{T}}\right)
\end{aligned}
$$

where $\mathcal{O}\left(C_{\mathbb{E}_{T}}\right)$ and $\mathcal{O}\left(C_{\mathcal{L}_{T}}\right)$ are respectively the computational complexity for $\mathbb{E}\left\{T_{i}\right\}$ in $(12)$ and $\mathcal{L}_{T_{i}}(\theta)$ in 15$)$. Note that numerical integrations are executed to compute $\mathbb{E}\left\{T_{i}\right\}$ and $\mathcal{L}_{T_{i}}(\theta)$, implying that $\mathcal{O}\left(C_{\mathbb{E}_{T}}\right)$ and $\mathcal{O}\left(C_{\mathcal{L}_{T}}\right)$ are large. The detailed complexity illustrations are presented in Appendix VIII-E

In the next section, we exploit Jensen's inequality to approximate these expressions to their alternative closed-forms, in order that the computational complexity can be greatly reduced. Then the optimization problem is further illustrated and determined.

\section{Problem Relaxation AND ILlustration}

\section{A. Performance Bounds}

As can be noticed from (12), it is mathematically intractable to derive the exact closed-form expression of $\mathbb{E}\left\{T_{i}\right\}$. Fortunately, $\mathbb{E}\left\{T_{i}\right\}$ is strictly convex w.r.t. $x$. Using Jensen's inequality, the transmission rate is bounded as [34], [35]

$$
J \triangleq\left\{\begin{array}{l}
C_{i}\left(h_{i}\right) \leq B \log \left(1+\frac{p_{i}}{\sigma_{i}^{2}} \mathbb{E}\left\{X_{i}\right\}\right) \triangleq C_{i}^{(\text {upper })}\left(h_{i}\right) \\
C_{i}\left(h_{i}\right) \geq B \log \left(1+\frac{p_{i}}{\sigma_{i}^{2} \mathbb{E}\left\{\frac{1}{X_{i}}\right\}}\right) \triangleq C_{i}^{(\text {lower })}\left(h_{i}\right)
\end{array}\right.
$$

In practice, to guarantee the QoS of data transmissions, SNR at the receivers is usually high. Since $\log \left(1+x^{\prime}\right)$ tends to change subtly in the high $x^{\prime}$ regime, it follows that $C_{i}^{(\text {lower })}\left(h_{i}\right) \simeq C_{i}^{(\text {upper })}\left(h_{i}\right)$. Thereby, the expectation of $T_{i}$ can be approximated by

$$
\mathbb{E}\left\{T_{i}\right\} \simeq C_{i}^{(\text {upper })}\left(h_{i}\right) \triangleq \frac{l}{B \log \left(1+\frac{p_{i}}{\sigma_{i}^{2}} \sigma_{h_{i}}^{2}\right)} .
$$

Due to the irreducible integral computation in $(15)$, it is also intractable to derive the exact closed-form of $\mathcal{L}_{T_{i}}(\theta)$. Let $\nabla_{x}^{2}(\cdot)$ represent the second order derivative operator w.r.t. $x$. For the sake of clarity, we introduce $\varsigma(x)$ and define it as $\varsigma(x)=\exp \left(-\theta \frac{l}{C_{i}(x)}\right)$. Then, 15 can be rewritten as

$$
\mathcal{L}_{T_{i}}(\theta)=\int_{0}^{+\infty} \varsigma(x) g_{X_{i}}(x) d x .
$$

Note that $\theta \geq 0$ in our above formulae. After some simple calculations, we have $\nabla_{x}^{2}(\varsigma(x))<0$ in the high SNR region, indicating that $\varsigma(x)$ is concave w.r.t. $x$ in the high SNR regime. Additionally, from (51), we note that $\mathcal{L}_{T_{i}}(\theta)=\mathbb{E}\{\varsigma(x \mid \theta)\}$.
Similar to the approximation method in $50, \mathcal{L}_{T_{i}}(\theta)$ can be upper bounded by exploiting the concavity property, i.e.,

$$
\mathcal{L}_{T_{i}}(\theta) \leq \exp \left(-\theta \frac{l}{B \log \left(1+\frac{p_{i}}{\sigma_{i}^{2}} \sigma_{h_{i}}^{2}\right)}\right) .
$$

Following the similar approximation method, in the high SNR regime, $\mathcal{L}_{T_{i}}(\theta)$ can be approximated by

$$
\mathcal{L}_{T_{i}}(\theta) \simeq \exp \left(-\theta \frac{l}{B \log \left(1+\frac{p_{i}}{\sigma_{i}^{2}} \sigma_{h_{i}}^{2}\right)}\right) .
$$

With the above mathematical transforms, $\mathbb{E}\left\{T_{i}\right\}$ and $\mathcal{L}_{T_{i}}(\theta)$ are converted into exponential functions, which are tractable closed-form and can be very easily evaluated. More importantly, based on the aforementioned approximations, manifold integral calculations are avoided and the computational complexity is significantly reduced. We present the brief complexity analyses in what follows. Following the derivations in (48), we find that the lower bound of the complexity, denoted by $C_{\text {total }}^{\prime}$, is

$$
C_{\text {total }}^{\prime}=\mathcal{O}\left(c_{\mathbb{E}_{T}}+2 n(n+1) c_{\mathcal{L}_{T}}\right)
$$

where $\mathcal{O}\left(c_{\mathbb{E}_{T}}\right)$ and $\mathcal{O}\left(c_{\mathcal{L}_{T}}\right)$ respectively represent the complexity for computing (50) and 53). Compared with $\mathcal{O}\left(C_{\mathbb{E}_{T}}\right)$ and $\mathcal{O}\left(C_{\mathcal{L}_{T}}\right), \mathcal{O}\left(c_{\mathbb{E}_{T}}\right)$ and $\mathcal{O}\left(c_{\mathcal{L}_{T}}\right)$ are considerably small. Hence, the overall complexity is reduced with our approximation method.

\section{B. Problem Analysis and Determination}

In this following, we further illustrate that the determination of global optimal $N$ can be obtained numerically by analysing both the objective and mean delay constraint.

We first present how to derive the feasible range of $N$ that satisfies the mean delay constraint. Typically, a larger $N$ results in a longer queue length both in the idle and subcycle periods. Given that the data transmitting time on each individual link is fixed, a longer queue length implies that an end-user experiences more waiting time. Correspondingly, it can be concluded that $\mathbb{E}\left\{W_{q}\right\}$ monotonically increases with $N$, which will also be numerically verified in Subsection VI-B

The bisection method can be applied to obtain the maximum tolerable $N$, hereinafter denoted as $N_{\max }$, that adheres to the delay constraint (35). From (47), $N_{\max }$ is uniquely determined by

$$
\left.\mathbb{E}\left\{W_{q}\right\}\right|_{N=N_{\max }} \leq D_{0} \text {, and }\left.\mathbb{E}\left\{\mathrm{W}_{\mathrm{q}}\right\}\right|_{\mathrm{N}=\mathrm{N}_{\max }+1}>\mathrm{D}_{0} .
$$

In the following, we further explain that the global optimal $N$ can be obtained efficiently. Denote the optimum $N$ that minimizes $\mathbb{E}\left\{P_{t o t}\right\}$ as $N^{\prime}$. Clearly, the optimum solution of $\mathbf{P 1}$ is

$$
N^{*}=\min \left\{N_{\max }, N^{\prime}\right\} .
$$

To determine $N^{\prime}$, we first analyse the monotonicity property of $\mathbb{E}\left\{P_{t o t}\right\}$. The following three engineering insights and outcomes can be achieved: 
i) $\frac{\mathbb{E}_{u}^{\text {tot }}}{\mathbb{E}\left\{T_{R C}\right\}}$ can be rewritten as

$$
\begin{aligned}
\frac{\mathbb{E}_{u}^{\text {tot }}}{\mathbb{E}\left\{T_{R C}\right\}} & =\lambda \mathbb{E}\left\{T_{u}^{i d l e}\right\} \cdot P_{0, u}+\lambda \mathbb{E}\left\{T_{u}^{t}\right\} \cdot P_{T, u} \\
& =\lambda\left(\mathbb{E}\left\{W_{q}\right\}-\mathbb{E}\left\{T_{1}\right\}\right) \cdot P_{0, u}+\lambda \mathbb{E}\left\{T_{u}^{t}\right\} \cdot P_{T, u}
\end{aligned}
$$

$\mathbb{E}\left\{T_{1}\right\}$ and $\mathbb{E}\left\{T_{u}^{t}\right\}$ are constant w.r.t. $N$; and $\mathbb{E}\left\{W_{q}\right\}$ monotonically increases with $N$. In the case that $P_{0, u}$ takes a sufficiently small value, $\frac{\mathbb{E}_{u}^{\text {tot }}}{\mathbb{E}\left\{T_{R C}\right\}}$ increases very slightly with $N$; otherwise, it grows fast with $N$.

ii) $\pi_{0}$ decreases with $N$, resulting in a decreasing mode switching frequency and further a fall of $\frac{\mathbb{E}_{s w}^{t o t}}{\mathbb{E}\left\{T_{R C}\right\}}$. The illustration will be presented in subsection VI-A and demonstrated in Fig. 3 (b).

iii) $\frac{\mathbb{E}_{R}^{t}+\mathbb{E}_{A P}^{t o t}}{\mathbb{E}\left\{T_{R C}\right\}}$ can be calculated as

$$
\begin{aligned}
& \frac{\mathbb{E}_{R}^{\text {tot }}+\mathbb{E}_{A P}^{\text {tot }}}{\mathbb{E}\left\{T_{R C}\right\}} \stackrel{11,13}{\underbrace{\rho \cdot P_{\text {sleep }, R}+\lambda \mathbb{E}\left\{T_{1}\right\} \cdot P_{0, R}+\lambda \mathbb{E}\left\{T_{2}\right\} \cdot P_{T, R}}_{\text {Power consumption at the Relay }}} \\
& +\underbrace{\left(\rho+\lambda \mathbb{E}\left\{T_{1}\right\}\right) \cdot P_{\text {sleep, } A P}+\lambda \mathbb{E}\left\{T_{2}\right\} \cdot P_{0, A P}}_{\text {Power consumption at the } A P},
\end{aligned}
$$

where $\rho=1-\lambda \mathbb{E}\left\{T_{1}\right\}-\lambda \mathbb{E}\left\{T_{2}\right\}$. It can be observed that $\frac{\mathbb{E}_{R}^{t o t}+\mathbb{E}_{A P}^{t o t}}{\mathbb{E}\left\{T_{R C}\right\}}$ remains invariant w.r.t. $N$.

Based on the aforementioned analyses, it arises that there exists a fundamental trade-off between end-users' power consumption (i.e., $\frac{\mathbb{E}_{u}^{\text {tot }}}{\mathbb{E}\left\{T_{R C}\right\}}$ ) and mode transition power consumption (i.e., $\frac{\mathbb{E}_{s w}^{t o t}}{\mathbb{E}\left\{T_{R C}\right\}}$ ). The monotonicity of $\mathbb{E}\left\{P_{t o t}\right\}$ is hard to analyze due to the existence of $\pi_{0}$.

It is worthwhile to note that $N_{0}(z)$ converges for any $|z|<1$ as illustrated in Proposition 1. Then with the approximation method, tractable closed-formulae can be derived, easily evaluated, and hence the overall computational complexity in obtaining $N^{\prime}$ can be greatly reduced. The resulting table can be stored in the device, which will facilitate engineering designs.

\section{NUMERICAL RESUltS AND Discussions}

In this section, we seek to find the approximated optimum solutions and reveal the mean power consumption and waiting time trade-offs. The performance comparison between the approximation method and exact calculations will also be presented.

The parameters are given in Table III unless otherwise stated. Note that packets' transmitting power, i.e., $p_{1}$ and $p_{2}$ take their maximum values to diminish each packet's transmission time (equivalently, the FSS and SSS durations are reduced), aiming at saving the overall electrical circuit energy. To obtain insightful conclusions and facilitate the analysis, we normalize $\sigma_{h_{i}}$ and assume that $\frac{p_{1}}{\sigma_{1}^{2}}=\frac{p_{2}}{\sigma_{2}^{2}}$.

\section{A. Verification of the Theoretical Results}

$\mathbb{E}\left\{P_{t o t}\right\}$ and $\pi_{0}$ curves versus $N$ are respectively depicted in Figs. 3 (a) and (b). To verify the exact theoretical results and approximated closed-formulae, simulation results are also
TABLE III

PARAMETER SetTings

\begin{tabular}{||c|c||c|c||}
\hline Parameters & Value & Parameters & Value \\
\hline \hline$p_{1}=p_{2}$ & $0.1 \mathrm{~W}$ & $P_{0, R}=P_{0, A P}$ & $10 \mathrm{~W}[32]$ \\
\hline$\triangle P, R$ & $2.6\lceil 32]$ & $P_{\text {listen, }}=P_{\text {listen }, A P}$ & $4 \mathrm{~W}[32]$ \\
\hline$p_{i} / \sigma_{i}^{2}(i=1,2)$ & $40 \mathrm{~dB}$ & $B$ & $1 \mathrm{MHz}$ \\
\hline$P_{0, u}$ & $0.5 \mathrm{~W}$ & $E_{s w}$ & $8 \mathrm{Joules}$ \\
\hline$\triangle_{P, u}$ & 1.2 & $l$ & $10^{4} \mathrm{bits}$ \\
\hline$\sigma_{h_{1}}^{2}$ & 1 & $\sigma_{h_{2}}^{2}$ & 1 \\
\hline \multicolumn{2}{|c}{} \\
\hline
\end{tabular}

presented. Note that the exact theoretical results for the average power consumption are achieved from (46), where $\mathbb{E}\left\{T_{i}\right\}$ and $\mathcal{L}_{T_{i}}(\theta)$ are used and exactly calculated based on (12) and (14), respectively. However, to efficiently obtain the approximated theoretical results of the average power consumption, $\mathbb{E}\left\{T_{i}\right\}$ and $\mathcal{L}_{T_{i}}(\theta)$ are respectively approximated by 50 and 53. Additionally, the idle probability expression is presented in (21). To exactly calculate its value, $\mathcal{L}_{T_{i}}(\theta)$ as an intermediate parameter, is explicitly calculated based on (15). As a contrast, the approximated idle probability is calculated based on (53).

Additionally, simulations are performed by respectively averaging $\mathbb{E}\left\{P_{\text {tot }}\right\}$ and $\pi_{0}$ over $10^{7}$ realizations, including $10^{4}$ random realizations of Rayleigh fading channels (as the outer loop in the simulations) and $10^{3}$ consecutive FSSs and SSSs for each channel realization (as the inner loop in the simulations).

As can be seen, theoretical curves match well with the simulations. Furthermore, it can be noticed that Jensen's bounds in (50) and (53) are quite tight in the considered high SNR scenario. Additionally, we observe that, $\pi_{0}$ decreases with $N$, which is reasonable and agrees with the intuition. The underlying reason is that a larger $N$ indicates a longer data transmission time during the sub-service cycle and hence, more packets join the waiting queue and wait for their subservice cycle turn. As a consequence, there is a higher probability that $N_{2}>0$, indicating a smaller $\pi_{0}$ (recall that $\left.\pi_{0}=\operatorname{Pr}\left\{N_{2}=0\right\}\right)$.
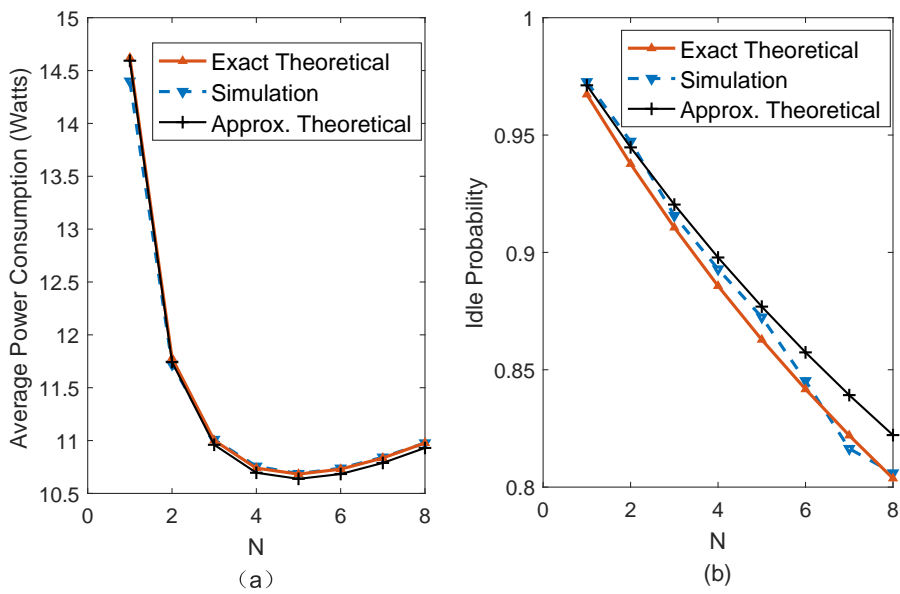

Fig. 3. Verification of the theoretical derivations; $\mathbb{E}\left\{P_{t o t}\right\}$ and $\pi_{0}$ versus $N$; $\lambda=0.2$.

It is worth noticing that though $N_{0}(\cdot)$ presented in 20, 
appears to include infinite terms; however, in the following, we numerically demonstrate that it converges at a fast rate under our proposed approach. We take the convergence property of $N_{0}\left(\mathcal{L}_{T_{2}}(\theta)\right)$ in (21) and (28) as an illustrative example. As indicated in Fig. 4. $N_{0}\left(\mathcal{L}_{T_{2}}(\theta)\right)$ converges when $n=7$ for various $\theta$ values. Note that $N_{0}\left(\mathcal{L}_{T_{2}}(0)\right) \equiv 1$, which matches with the theoretical results. This is because $\mathcal{L}_{T_{2}}(0) \equiv 1$ at $\theta=0$, then $N_{0}\left(\mathcal{L}_{T_{2}}(0)\right) \equiv 1$ can be achieved based on the property of the PGF function. Additionally, the approximated closed-forms in (50) and (53) are also considered. The curves reveal that in the high SNR scenario, the approximated approach behaves quite similar as the exact formulae.

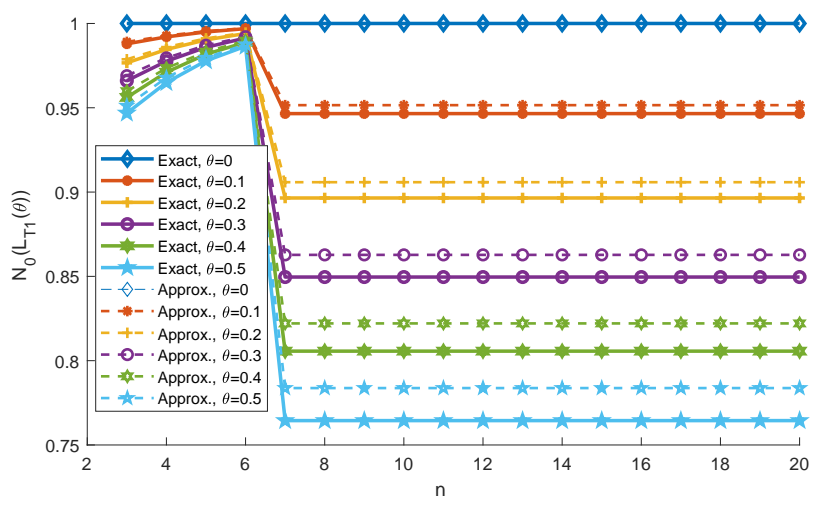

Fig. 4. The convergence curves of $N_{0}\left(\mathcal{L}_{T_{1}}(\theta)\right)$ versus $n ; \lambda=0.2$.

\section{B. Impact of $N$}

To investigate the impacts of $N$ on $\mathbb{E}\left\{P_{\text {tot }}\right\}$ and $\mathbb{E}\left\{W_{q}\right\}$, we plot $\mathbb{E}\left\{P_{\text {tot }}\right\}$ and $\mathbb{E}\left\{W_{q}\right\}$ curved surfaces in Figs. 5 and 6 . It can be noticed that $\mathbb{E}\left\{P_{\text {tot }}\right\}$ first decreases with $N$ before achieving its minimum value, and afterwards grows with $N$. Besides, as demonstrated in Fig. 6, $\mathbb{E}\left\{W_{q}\right\}$ monotonically increases with $N$. Therefore, sacrificing the mean waiting time not necessarily brings the benefit of power savings. The underlying reason is analyzed as follows.

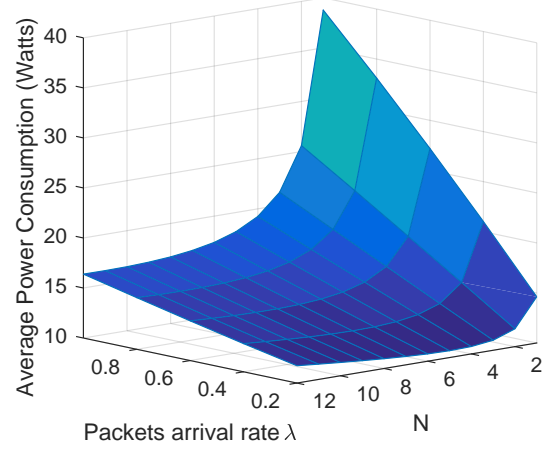

Fig. 5. $\mathbb{E}\left\{P_{\text {tot }}\right\}$ versus $\lambda$ and $N$.

Firstly, in the small $N$ region, the mean waiting time and $\mathbb{E}\left\{T_{u}^{i d l e}\right\}$ are small, which results in a small $\mathbb{E}_{u}^{\text {tot }}$ value. Thereby, $\mathbb{E}\left\{P_{\text {tot }}\right\}$ is dominated by the power consumed at the AP and relay server. Secondly, as illustrated in subsection VI-A and demonstrated in Fig. 3 (b), $\pi_{0}$ decreases with $N$, leading to a decreasing mode switching frequency and $\frac{\mathbb{E}_{s w}^{t o t}}{\mathbb{E}\left\{T_{R C}\right\}}$. Thirdly, after some simple calculations, it can be revealed that $\frac{\mathbb{E}_{R}^{t o t}+\mathbb{E}_{A P}^{t o t}}{\mathbb{E}\left\{T_{R C}\right\}}$ in 46 keeps constant w.r.t. $N$. Based on the aforementioned analysis, we claim that the total power consumed at the AP and relay server, i.e., $\left(\frac{\mathbb{E}_{s w}^{t o t}}{\mathbb{E}\left\{T_{R C}\right\}}+\frac{\mathbb{E}_{R}^{t o t}+\mathbb{E}_{A P}^{t o t}}{\mathbb{E}\left\{T_{R C}\right\}}\right)$ decreases with $N$.

However, as $N$ (when $N \geq N^{*}$ ) further increases, the mean waiting time grows significantly, as demonstrated in Fig. 6. Different from the small $N$ case, $\mathbb{E}\left\{T_{u}^{i d l e}\right\}$ takes a larger value as indicated in (38). Further, as can be observed from (57), increasing $\mathbb{E}\left\{T_{u}^{i d l e}\right\}$ results in a growing $\mathbb{E}_{u}^{\text {tot }}$ such that $\frac{\mathbb{E}_{u}^{\tau d t}}{\mathbb{E}\left\{T_{R C}\right\}}$ dominates the total power consumption instead of $\frac{\mathbb{E}_{s w}^{t o t}}{\mathbb{E}\left\{T_{R C}\right\}}$. The above finally leads to an increasing $\mathbb{E}\left\{P_{t o t}\right\}$.

\section{Impact of $\lambda$}

In the sequel, we study the impacts of $\lambda$ on $\mathbb{E}\left\{P_{\text {tot }}\right\}$ and $\mathbb{E}\left\{W_{q}\right\}$. In Figs. 5 and $6, \mathbb{E}\left\{P_{\text {tot }}\right\}$ and $\mathbb{E}\left\{W_{q}\right\}$ versus $\lambda$ are plotted, where $\lambda$ varies from 0.2 to 5 . Besides, in Fig. 8 (b), the optimal $\mathbb{E}\left\{P_{\text {tot }}\right\}$ curves versus $\lambda$ are also presented, where $\frac{p_{1}}{\sigma_{1}}=\frac{p_{2}}{\sigma_{2}}=40,60 \mathrm{~dB}$, respectively. We have the following two observations.

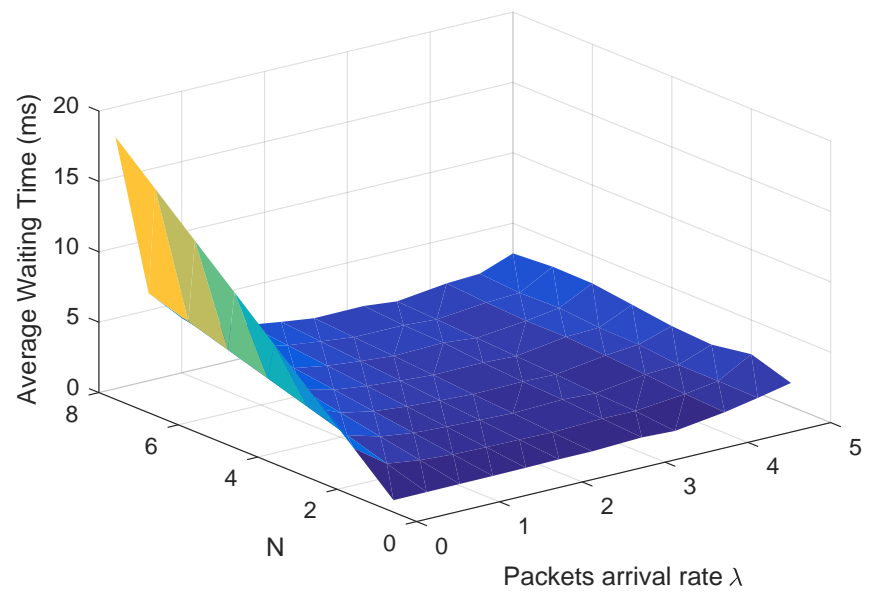

Fig. 6. $\mathbb{E}\left\{W_{q}\right\}$ surface versus $\lambda$ and $N$.

Firstly, the theoretical results show that $\mathbb{E}\left\{P_{\text {tot }}\right\}$ increases with $\lambda$. These advocate the intuitions and further verify the validity of our derivations. We first analyse the underlying reason that $\mathbb{E}\left\{P_{\text {tot }}\right\}$ increases with $\lambda$. A higher arrival rate means that the relays and AP are suffering a heavier traffic load, thereby consuming more power. Also, from the users' standpoint, more users are served per unit of time, resulting in higher power consumption. We further illustrate why $\mathbb{E}\left\{W_{q}\right\}$ first decreases and then increases with $\lambda$. As $\lambda$ increases from a small value, the queue length can easily arrive at the threshold. Hence, the FSS starts in a shorter time, leading to a decreasing $\mathbb{E}\left\{W_{q}\right\}$. As $\lambda$ further increases, the queue length becomes larger, and FSS and SSS last longer. Correspondingly, it takes a longer time for the user to be served, even though the FSS has started.

In Fig. 7, we present the tradeoffs between the average power consumption $\mathbb{E}\left\{P_{t o t}\right\}$ and $D_{0}$ when $\lambda=0.2,0.4$, and 0.6 , respectively. For the scheme without optimization 
(or "non-Opt" for short), $N=N_{\max }$ (see (55)). Note that under the non-Opt scheme, $\mathbb{E}\left\{P_{t o t}\right\}$ first sharply decreases with $D_{0}$ and then gently increases. For the optimized results, $\mathbb{E}\left\{P_{t o t}\right\}$ does not increase with $D_{0}$. It means that our scheme guarantees that sacrificing the delay will bring power saving gains.

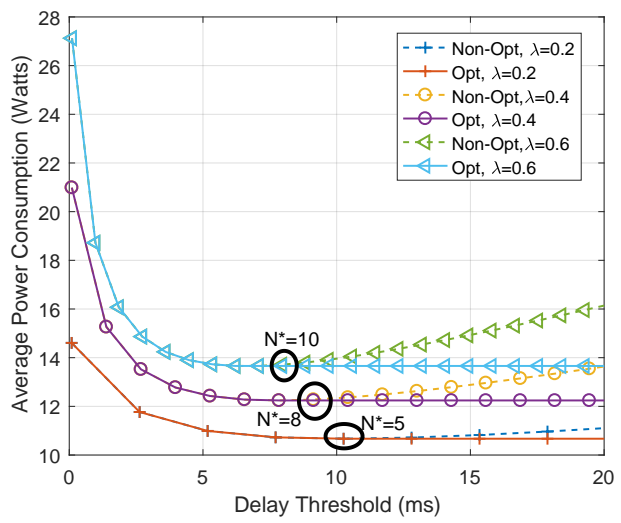

Fig. 7. Tradeoff between the power consumption and mean waiting time; $D_{0}=20 \mathrm{~ms}$.

Additionally, from Fig. 7, we notice that in a denser packet arrival scenario, the energy-saving service threshold takes a larger value. This is because a larger $\lambda$ implies a smaller packet inter-arrival and shorter time span to be taken to accumulate a certain number of packets. Hence, for a given mean waiting time tolerance $D_{0}, N$ is allowed to take a larger value compared with the smaller $\lambda$ scenario. For a certain mean waiting time, according to (57), a larger $N$ does not result in a larger $\frac{\mathbb{E}_{u}^{\text {tot }}}{\mathbb{E}\left\{T_{R C}\right\}}$ than that of the smaller $\lambda$ scenario. Meanwhile, based on the analyses in $\mathrm{V}-\mathrm{B}$ ii) and iii), a larger $N$ leads to a lower $\frac{\mathbb{E}_{R}^{t o t}+\mathbb{E}_{A P}^{t o t}}{\mathbb{E}\left\{T_{R C}\right\}}$. To rap, $N^{*}$ takes a larger value in the denser packet arrival scenario.

\section{Performance Comparison with Always-on-Service Policy}

In Figs. 8 (a) and (b), the average power consumption of the optimum-threshold policy is compared with that of the Always-on-Service (AoS) policy [26], where the relay server is activated once one packet arrives. It is clear that the optimal (OP) policy performs better than the AoS policy in terms of the average power consumption.

Additionally, it is demonstrated that for the optimal policy, a larger maximum transmitting power (corresponding to a higher $\left.p_{i} / \sigma_{i}, \forall i \in\{1,2\}\right)$ results in reduced average power consumption. This is due to the fact that a larger $p_{i} / \sigma_{i}$ $(\forall i \in\{1,2\})$ results in a larger transmitting rate and further a reduced service time span. Overall power consumption at the relay server and the AP correspondingly decreases.

Furthermore, numerical results obtained from the approximated closed-form are also presented. The curves in Fig. 8 (b) reveal that the approximated formulae get closer to the exact theoretical derivations as SNR increases. This coincides with the derivations in (49)-(53).

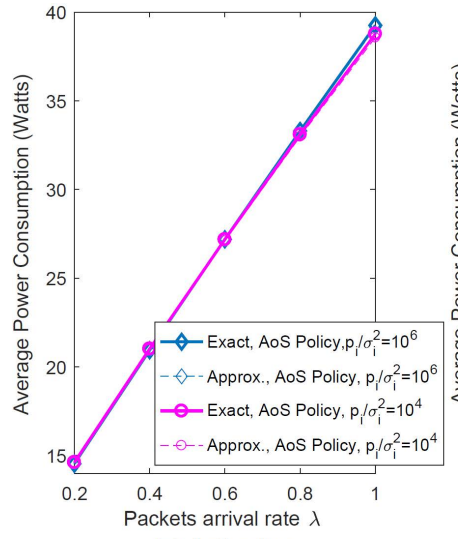

(a) Aos policy

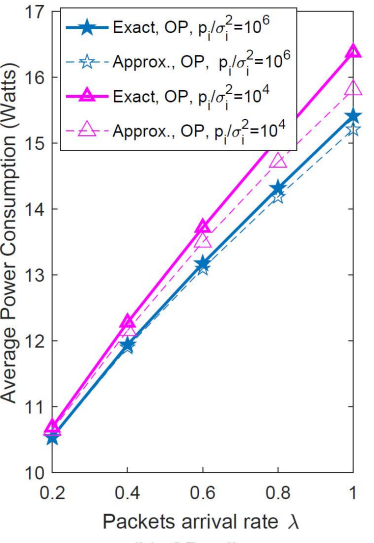

(b) OP policy
Fig. 8. $\mathbb{E}\left\{P_{\text {tot }}\right\}$ versus $\lambda$ under two different policies; $D_{0} \leq 10.5 \mathrm{~ms}$.

\section{CONCLUSIONS}

A two-stage, $N$-threshold and gated M/G/1 queueing communication has been proposed and investigated for the delaytolerant networks. Two important performance metrics, including the mean waiting time and long-term expected power consumption have been analysed and associated with packet arrival rate, relay service threshold as well as channel statistics. A more practical expected power consumption was derived in the sense that it included the electrical circuit energy consumption. The expected power consumption minimization problem has been formulated under the mean delay constraint. Mathematical approximation methods based on Jensen's inequality were adopted to obtain the tight closed-form bounds, such that computational complexity can be greatly reduced. The above studies and mathematical propositions will provide guides for practical system design. The numerical results illustrated the suitability of Jensen's bounds and fast convergence rate of closed-formulae. The tradeoff between the mean waiting time and power consumptions are revealed. Additionally, it has been revealed that: 1) in a larger packet arrival rate scenario, the energy-saving service threshold takes a larger value; 2) performance comparison with the always-on-service policy demonstrates the advantage of our proposed scheme.

\section{APPENDIX}

\section{A. Derivations of (9) and (13)}

The total time it takes the relay to serve $\Gamma$ packets in the FSSs and SSSs are respectively $T_{1,1}+T_{1,2}+\cdots+T_{1, \Gamma}$ and $T_{2,1}+T_{2,2}+\cdots+T_{2, \Gamma}$ seconds. Clearly, $\Gamma, T_{1, i}$, and $T_{2, i}$ are all random variables. $T_{j, 1}, T_{j, 2}, \cdots, T_{j, \Gamma}(j=1,2)$ are identically distributed. According to Wald's equation, the following equation holds:

$$
\mathbb{E}\left\{T_{i, 1}+T_{i, 2}+\cdots+T_{i, \Gamma}\right\}=\mathbb{E}\{\Gamma\} \mathbb{E}\left\{T_{i}\right\}, i=1,2 .
$$

Then based on the delay cycle property in Section 1.2 of [23], we have (9). Further, note that

$$
\begin{aligned}
T_{R C}= & T_{0}+T_{1,1}+T_{1,2}+\cdots+T_{1, \Gamma} \\
& +T_{2,1}+T_{2,2}+\cdots+T_{2, \Gamma} .
\end{aligned}
$$


By combining (60) with (59), we have

$$
\begin{aligned}
& \mathbb{E}\left\{T_{R C}\right\}=\mathbb{E}\left\{T_{0}\right\}+\mathbb{E}\{\Gamma\} \mathbb{E}\left\{T_{1}\right\}+\mathbb{E}\{\Gamma\} \mathbb{E}\left\{T_{2}\right\}
\end{aligned}
$$

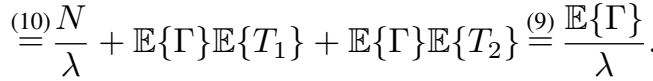

\section{B. Proof of Proposition 1}

The following notations are defined:

$$
\begin{aligned}
g_{n}(z) & =\pi_{0} \eta\left(f_{n}(z)\right), n \in \mathbb{N} \\
\eta(z) & =1-z^{N} \\
f_{0}(z) & =z \\
f_{1}(z) & =\mathcal{L}_{T_{2}}(\lambda-\lambda z), \\
f_{n}(z)=f_{n-1}\left(f_{1}(z)\right) & =f_{1}\left(f_{n-1}(z)\right), n \in \mathbb{N}^{+}, n \geq 2
\end{aligned}
$$

Under the assumption that $T_{1}$ and $T_{2}$ have the same distribution, then

$$
f_{T_{1}}(t)=f_{T_{2}}(t) \text {, and } \mathcal{L}_{\mathrm{T}_{1}}(\mathrm{z})=\mathcal{L}_{\mathrm{T}_{2}}(\mathrm{z}) .
$$

According to (17)-(19), we have

$$
\begin{aligned}
& N_{0}(z) \stackrel{19}{=} N_{0}\left(\mathcal{L}_{T_{2}}(\lambda-\lambda z)\right) N_{0}\left(\mathcal{L}_{T_{1}}(\lambda-\lambda z)\right)-\pi_{0} \eta(z) \\
& \stackrel{(a)}{=} N_{0}\left(\mathcal{L}_{T_{2}}(\lambda-\lambda z)\right) N_{0}\left(\mathcal{L}_{T_{2}}(\lambda-\lambda z)\right)-\pi_{0} \eta(z) \\
& \stackrel{(b)}{=}\left\{N_{0}\left(f_{1}\right)\right\}^{2}-g_{0}(z), \\
& \stackrel{(c)}{=}\left(\left[\left(\left\{N_{0}\left(f_{n}\right)\right\}^{2}-g_{n-1}(z)\right)^{2}-g_{n-2}(z)\right]^{2}-\cdots\right. \\
& \left.-g_{1}(z)\right)^{2}-g_{0}(z), n \rightarrow \infty \\
& \stackrel{(d)}{=} 20) \text {, }
\end{aligned}
$$

where $(a)$ is achieved from 677; $(b)$ is based on 667; $(c)$ is obtained by successively substituting (70) into (68); $(d)$ satisfies with the fact that for any $z$ with $|z|<1$ [22],

$$
f_{n}(z) \rightarrow z_{\infty} \equiv 1, \text { as } n \rightarrow \infty .
$$

Further, we have

$$
N_{0}\left(f_{n}\right) \rightarrow 1, \text { and } \mathrm{g}_{\mathrm{n}}(\mathrm{z}) \rightarrow 0, \text { as } \mathrm{n} \rightarrow \infty .
$$

We further illustrate that $N_{0}(z)$ converges absolutely for any $|z|<1$. When $z=1, f_{n}(1) \equiv 1(\forall n)$ can be obtained from the recursion formula in (66). Further, from (62), it follows that $g_{n}(z)=0(\forall n)$ when $z=1$. Then, it can be observed that $N_{0}(1)=1$ from 20 . According to the property of PGF [23], the convergence of $N_{0}(z)$ for any $|z|<1$ is proved.

\section{Proof of Proposition 2}

By substituting $z=0$ into 69, we have

$$
\begin{gathered}
N_{0}(0)=\left\{N_{0}\left(\mathcal{L}_{T_{2}}(\lambda)\right)\right\}^{2}-\pi_{0} \\
\stackrel{(i)}{=} \operatorname{Pr}\left\{N_{0}=0\right\} \stackrel{(e)}{=} 0,
\end{gathered}
$$

where $(i)$ is based on the property of p.d.f. function; $(e)$ satisfies with the fact that $N_{0}$ is strictly positive, as indicated in (1).

Hence, we have $\pi_{0}=\left\{N_{0}\left(\mathcal{L}_{T_{2}}(\lambda)\right)\right\}^{2}$.

\section{Proof of Equation 36}

Proof. For easing notations, in the following, we introduce a parameter $f_{k}(\theta)$ as follows:

$$
f_{k}(\theta)=W_{q}(\theta \mid \text { case } k) \cdot \operatorname{Pr}\{\text { Case } k\}, \forall k \in\{1,2,3\} .
$$

In the following, the proof is performed according to the property of the LST.

For a continuous random variable (referred to as $X$ ) with cumulative distribution function $F(t)$, the moments of $X$ can be computed using [31] $\mathrm{E}\left[X^{n}\right]=\left.(-1)^{n} \frac{\mathrm{d}^{n}\left\{\mathcal{L}_{F}(\theta)\right\}}{\mathrm{d} \theta^{n}}\right|_{\theta=0}$, where $\mathcal{L}_{F}(\theta)$ is the Laplace-Stieltjes transforms of $F(t)$.

when $n=1$, we have $\mathrm{E}[X]=-\left.\frac{\mathrm{d}\left\{\mathcal{L}_{F}(\theta)\right\}}{\mathrm{d} \theta}\right|_{\theta=0}$.

Following the property, we have

$$
\mathbb{E}\left\{W_{q}\right\}=-\left.\nabla_{\theta}\left(f_{1}(\theta) \cdot f_{2}(\theta) \cdot f_{3}(\theta)\right)\right|_{\theta=0},
$$

which can be rewritten as

$$
\begin{aligned}
\mathbb{E}\left\{W_{q}\right\}= & -\left.\nabla_{\theta}\left(f_{1}(\theta) \cdot f_{2}(\theta) \cdot f_{3}(\theta)\right)\right|_{\theta=0} \\
= & -\left.\nabla_{\theta}\left(f_{1}(\theta)\right)\right|_{\theta=0} \cdot f_{2}(0) \cdot f_{3}(0) \\
& -\left.\nabla_{\theta}\left(f_{2}(0)\right)\right|_{\theta=0} \cdot f_{1}(0) \cdot f_{3}(0) \\
& -\left.\nabla_{\theta}\left(f_{3}(0)\right)\right|_{\theta=0} \cdot f_{2}(0) \cdot f_{1}(0) .
\end{aligned}
$$

Since $f_{k}(0)=1, \forall k \in\{1,2,3\}$, (73) can be reduced into (36.

\section{E. Computational complexity analyses}

As can be observed from (22)-(30), the computational complexity of $W_{q}(\theta \mid$ case $j),(j=1,2, \cdots, 3)$ is mainly determined by $N_{0}\left(\mathcal{L}_{T_{i}}(\theta)\right), N_{0}\left(\mathcal{L}_{T_{i}}\left(\lambda-\lambda \mathcal{L}_{T_{j}}(\theta)\right)\right),(i, j=$ $1,2), \mathcal{L}_{T_{i}}(\theta)$, and $\mathbb{E}\left\{T^{\prime}\right\}$. Since the value of $\mathcal{L}_{T_{i}}(\theta)$ can be stored and used in the subsequent calculations, we only take its computational complexity into account once. Further, note that $\mathbb{E}\left\{T^{\prime}\right\}$ can be obtained from $N_{0}\left(\mathcal{L}_{T_{i}}(\theta)\right)$, the computational complexity of $\mathbb{E}\left\{T^{\prime}\right\}$ is also ignored. In the following, we first analyse computational complexity for $N_{0}\left(\mathcal{L}_{T_{i}}(\theta)\right)$.

According to the recursive derivation equations for $N_{0}\left(\mathcal{L}_{T_{i}}(\theta)\right)$, namely (62)-(71), we find that the computational complexity for $N_{0}\left(\mathcal{L}_{T_{i}}(\theta)\right)$, denoted by $C_{c}\left(N_{0}\left(\mathcal{L}_{T_{i}}(\theta)\right)\right)$, is determined by $C_{c}\left(f_{i}\left(\mathcal{L}_{T_{i}}(\theta)\right)\right),(i=0,1, \ldots, n-1)$. 
Note that $C_{c}\left(f_{1}\left(\mathcal{L}_{T_{i}}(\theta)\right)\right)=\mathcal{O}\left(C_{\mathcal{L}_{T}}\right)$. Further, based on the recursive derivations, we have the general formula as $C_{c}\left(f_{n}\left(\mathcal{L}_{T_{i}}(\theta)\right)\right)=\mathcal{O}\left(n C_{\mathcal{L}_{T}}\right)$. As a result,

$$
\begin{aligned}
C_{c}\left(N_{0}\left(\mathcal{L}_{T_{i}}(\theta)\right)\right) & =\mathcal{O}\left((1+2+\cdots+n) \cdot C_{\mathcal{L}_{T}}\right) \\
& =\mathcal{O}\left(\frac{n(n+1)}{2} C_{\mathcal{L}_{T}}\right) .
\end{aligned}
$$

Further, following the similar analyses, the computational complexity of $N_{0}\left(\mathcal{L}_{T_{i}}\left(\lambda-\lambda \mathcal{L}_{T_{j}}(\theta)\right)\right)$ can be written as follows:

$$
\begin{aligned}
C_{c}\left(N_{0}\left(\mathcal{L}_{T_{i}}\left(\lambda-\lambda \mathcal{L}_{T_{j}}(\theta)\right)\right)\right) & =\mathcal{O}\left((1+2+\cdots+n) \cdot 2 C_{\mathcal{L}_{T}}\right) \\
& =\mathcal{O}\left(n(n+1) C_{\mathcal{L}_{T}}\right) .
\end{aligned}
$$

Following the derivations of (74), the computational complexity for $\pi_{0}$ can be achieved as $\mathcal{O}\left(\frac{n(n+1)}{2} C_{\mathcal{L}_{T}}\right)$. Finally, (48) can be obtained.

\section{REFERENCES}

[1] Nan Qi, Nikolaos I. Miridakis, Theodoros A. Tsiftsis, and Rugui Yao, "On the Fundamental Queue Analysis for Relay-assisted Two-stage Communication Networks" IEEE WCNC 2019, to be published.

[2] J. G. Andrews, et al., "What Will 5G Be?" IEEE J. Sel. Areas Commun., vol. 32, no. 6, pp. 1065-1082, Jun. 2014.

[3] B. Rossi, "Gartner's Internet of Things predictions-Information Age," 2015. [Online]. Available: information-age.com/technology/mobile-andnetwork-ing/123458905/gartners-internet-things-predictions.

[4] W. Guo, S. Zhou, Y. Chen, S. Wang, X. Chu and Z. Niu, "Simultaneous Information and Energy Flow for IoT Relay Systems with Crowd Harvesting," IEEE Commun. Mag., vol. 54, no. 11, pp. 143-149, Nov. 2016.

[5] A. Manzoor, P. Porambage, M. Liyanage, M. Ylianttila and A. Gurtov, "DEMO: Mobile Relay Architecture for Low-Power IoT Devices," 2018 IEEE 19th International Symposium on WoWMoM), pp. 14-16, 2018.

[6] Kota Sastri and Giambene Giovanni, "Satellite 5G: IoT Use Case for Rural Areas Applications," in The Eleventh International Conference on Advances in Satellite and Space Communications - SPACOMM, 2019.

[7] T. Mekkawy, R. Yao, N. Qi, and Y. Lu, "Secure Relay Selection for Two Way Amplify-and-Forward Untrusted Relaying Networks?" IEEE Trans. Veh. Technol., 2018, [Online]. Available: https://ieeexplore.ieee.org/document/8502793/

[8] I. L. C. Araujo and A. Klautau, "Traffic-aware sleep mode algorithm for 5G networks," 2015 International Workshop on Telecommunications (IWT), pp. 1-5, 2015.

[9] I. Ashraf, F. Boccardi, and L. Ho, "Sleep mode techniques for small cell deployments," IEEE Commun. Mag., vol. 49, no. 8, pp. 7279, Aug. 2011.

[10] Y. Zguira, H. Rivano, A. Meddeb, "IoB-DTN: a lightweight DTN protocol for mobile IoT Applications to smart bike sharing systems," IEEE Wireless Days, pp. 131-136, 2018.

[11] F. M. Al-Turjman, A. E. Al-Fagih, W. M. Alsalih, and H. S. Hassanein, "A delay-tolerant framework for integrated RSNs in IoT," Comput. Commun., vol. 36, no. 9, pp. 998-1010, 2013.

[12] A. Elmangoush, R. Steinke, M. Catalan, A. Corici, T. Magedanz, and J. Oller, "Interconnecting Standard M2M Platforms to Delay Tolerant Networks," in 2nd International Conference on Future Internet of Things and Cloud (FiCloud 2014), pp. 258-263, Aug. 2014.

[13] A. Alsharoa, X. Zhang, D. Qiao and A. Kamal, "An Energy-Efficient Relaying Scheme for Internet of Things Communications," in IEEE International Conference on Communications (ICC), pp. 1-6, 2018.
[14] D. Djenouri and M. Bagaa, "Energy-aware constrained relay node deployment for sustainable wireless sensor networks," IEEE Trans. on Sustainable Comput., vol. 2, no. 1, pp. 30-42, Jan. 2017.

[15] C. Ma, W. Liang, and M. Zheng, "Set-covering-based algorithm for delay constrained relay node placement in wireless sensor networks," in IEEE International Conference on Communications (ICC), pp. 1-6, 2016.

[16] J. Kim, H. Lee and S. Chong, "Traffic-Aware Energy-Saving Base Station Sleeping and Clustering in Cooperative Networks," IEEE Trans. Wireless Commun., vol. 17, no. 2, pp. 1173-1186, Feb. 2018.

[17] E. N. Almeida, R. Campos and M. Ricardo, "Traffic-aware multi-tier flying network: Network planning for throughput improvement," 2018 IEEE Wireless Communications and Networking Conference (WCNC), Barcelona, pp. 1-6, 2018.

[18] L. Saker, S. E. Elayoubi, R. Combes, and T. Chahed, "Optimal control of wake up mechanisms of femtocells in heterogeneous networks," IEEE J. Sel. Areas Commun., vol. 30, no. 3, pp. 664-672, Apr. 2012.

[19] Z. Jiang, B. Krishnamachari, S. Zhou and Z. Niu, "Optimal Sleeping Mechanism for Multiple Servers With MMPP-Based Bursty Traffic Arrival," IEEE Wireless Commun. Lett., vol. 7, no. 3, pp. 436-439, Jun. 2018.

[20] J. Wu, Y. Bao, G. Miao, S. Zhou and Z. Niu, "Base-Station Sleeping Control and Power Matching for EnergyDelay Tradeoffs With Bursty Traffic," IEEE Trans. Veh. Technol., vol. 65, no. 5, pp. 3657-3675, May 2016.

[21] J. Wu, S. Zhou and Z. Niu, "Traffic-Aware Base Station Sleeping Control and Power Matching for Energy-Delay Tradeoffs in Green Cellular Networks," IEEE Trans. Wireless Commun., vol. 12, no. 8, pp. 41964209, Aug. 2013.

[22] B. Doshi, "Analysis of a two phase queueing system with general service times," Operations Research Letters, vol. 10, no. 5, pp. 265-272, 1991.

[23] H. Takagi, Queueing Analysis: A Foundation of Performance Evaluation, vol. 1. North-Holland. Amsterdam, 1991.

[24] Jiyeon Lee, Jongwoo Kim, "A workload-dependent M/G/1 queue under a two-stage service policy," Operations Research Letters, vol. 34, no. 5 , pp: 531-538, 2006.

[25] T. S. Kim, and H. M. Park, "Cycle analysis of a two-phase queueing model with threshold," European Journal of Operational Research, vol. 144 , no. 1 , pp. $157-165,2003$.

[26] J. Li, Y. Q. Zhao, F. R. Yu and X. Huang, "Queuing Analysis of TwoHop Relay Technology in LTE/LTE-A Networks With Unsaturated and Asymmetric Traffic," IEEE Internet of Things Journal, vol. 3, no. 3, pp. 378-385, Jun. 2016

[27] A. Wald, "On cumulative sums of random variables," The Annals of Mathematical Statistics, vol. 15, no.3, pp.283-296, 1944.

[28] D. Friesel, M. Buschhoff and O. Spinczyk, "Parameter-Aware Energy Models for Embedded-System Peripherals," in Proc. 2018 IEEE 13th International Symposium on Industrial Embedded Systems (SIES), 2018, pp. 1-4.

[29] A. Papoulis, S. U. Pillai, Probability random variables and stochastic processes, McGraw-Hill Higher Education, 2002.

[30] R. W. Wolff, "Poisson Arrivals See Time Averages," Operations Research, vol. 30, No. 2, pp. 157-165, Apr. 1982.

[31] M. Harchol-Balter, "Transform Analysis" in Performance Modeling and Design of Computer Systems: Queueing Theory in Action, Cambridge: Cambridge University Press, 2012, pp. 433-449.

[32] G. Auer, V. Giannini, I. Godor, P. Skillermark, M. Olsson, M. A. Imran, D. Sabella, M. J. Gonzalez, O. Blume and A. Fehske, "How much energy is needed to run a wireless network?" IEEE Trans. Wireless Commun., vol. 18, pp. 40-49, Oct. 2011.

[33] I. N. Bronstein, K. A. Semendjajew, G. Musiol, Taschenbuch der mathematik, Springer-Verlag, vol. 1, pp. 617-620, 2012.

[34] J. Yuan, M. Matthaiou, S. Jin and F. Gao, "Tightness of Jensen's Bounds and Applications to MIMO Communications," IEEE Trans. Commun., vol. 65, no. 2, pp. 579-593, Feb. 2017.

[35] S. Zhang, Y. Wang, Z. He and S. Jin, "Ergodic rate analysis on applying antenna selection in D2D communication underlaying cellular networks," China Communications, vol. 14, no. 11, pp. 167-184, Nov. 2017. 